\title{
Mortality Monitoring Design for Utility-Scale Solar Power Facilities
}

Open-File Report 2016-1087 



\section{Mortality Monitoring Design for Utility-Scale Solar Power Facilities}

By Manuela Huso, Thomas Dietsch, and Chris Nicolai

Prepared in cooperation with the U.S. Fish and Wildlife Service

Open-File Report 2016-1087

U.S. Department of the Interior

U.S. Geological Survey 


\section{U.S. Department of the Interior \\ SALLY JEWELL, Secretary}

\section{U.S. Geological Survey \\ Suzette M. Kimball, Director}

U.S. Geological Survey, Reston, Virginia: 2016

For more information on the USGS-the Federal source for science about the Earth,

its natural and living resources, natural hazards, and the environment-visit

http://www.usgs.gov/ or call 1-888-ASK-USGS (1-888-275-8747).

For an overview of USGS information products, including maps, imagery, and publications, visit http://store.usgs.gov/.

Findings and conclusions in this article are those of the authors and do not necessarily represent the views of the U.S. Fish and Wildlife Service.

All photographs were taken by the authors unless otherwise noted.

Any use of trade, firm, or product names is for descriptive purposes only and does not imply endorsement by the U.S. Government.

Although this information product, for the most part, is in the public domain, it also may contain copyrighted materials as noted in the text. Permission to reproduce copyrighted items must be secured from the copyright owner.

Suggested citation:

Huso, Manuela, Dietsch, Thomas, and Nicolai, Chris, 2016, Mortality monitoring design for utility-scale solar power facilities: U.S. Geological Survey Open-File Report 2016-1087, 44 p., http://dx.doi.org/10.3133/ofr20161087.

ISSN 2331-1258 


\section{Contents}

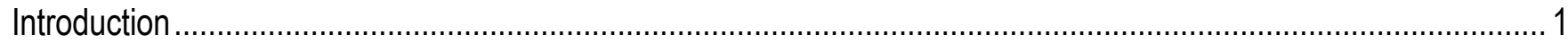

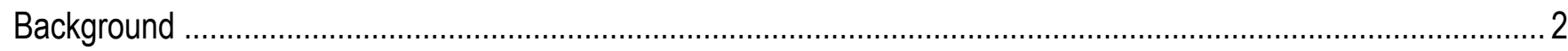

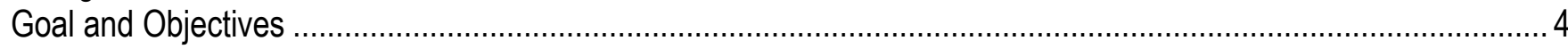

Objective 1 - Monitoring to Estimate Total Bird and Bat Mortality................................................................. 4

Objective 2 - Monitoring to Determine Spatial or Temporal/Seasonal Patterns of Total Bird Mortality ................. 5

Objective 3 - Monitoring to Evaluate Which Species and Taxonomic Groups Are at Risk..................................5

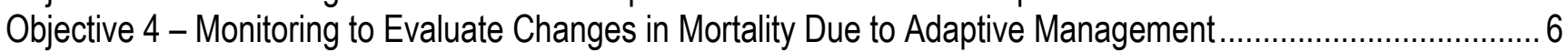

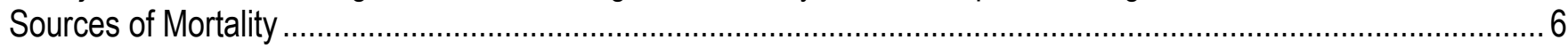

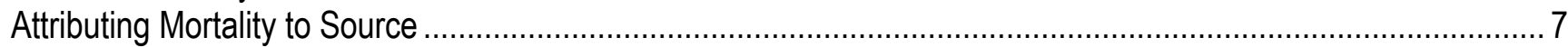

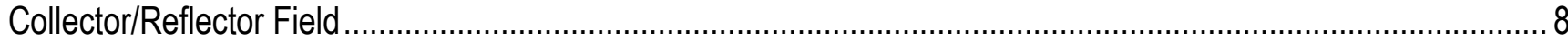

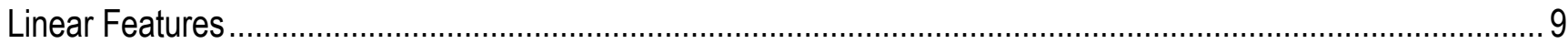

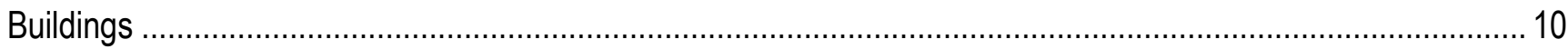

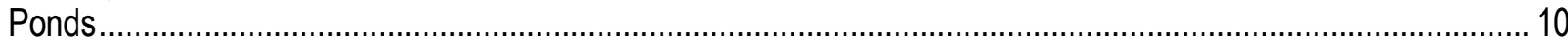

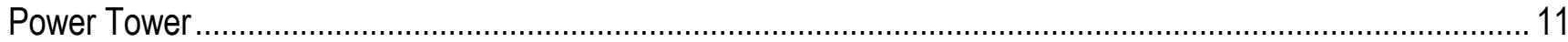

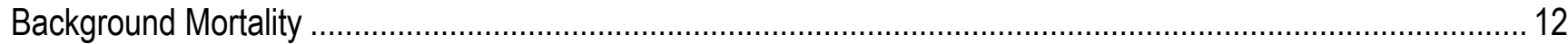

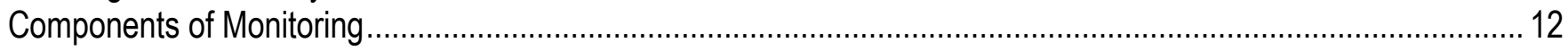

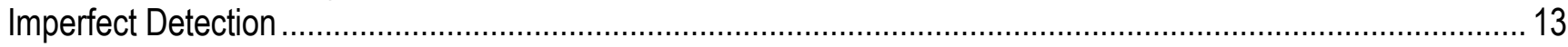

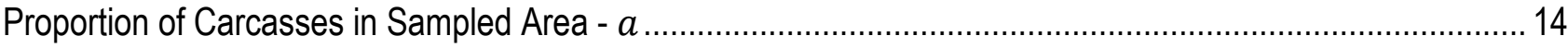

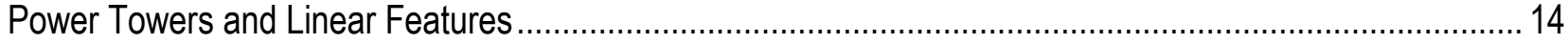

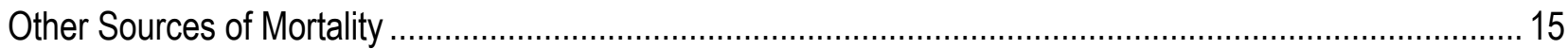

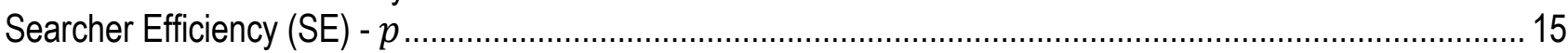

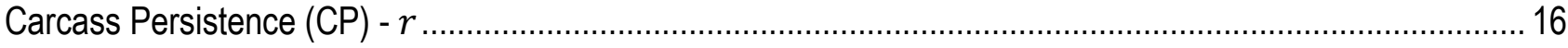

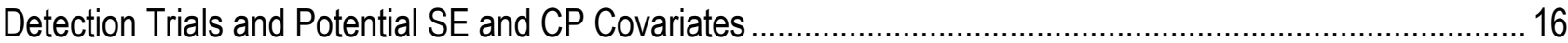

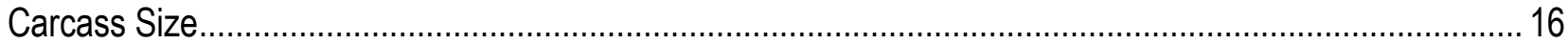

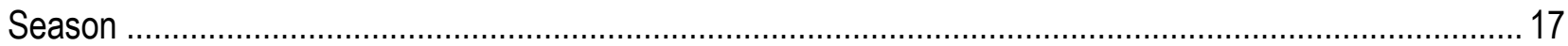

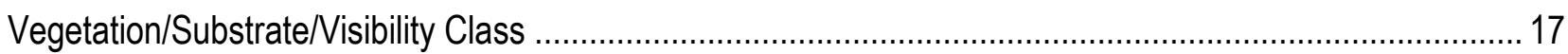

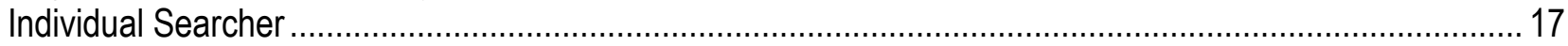

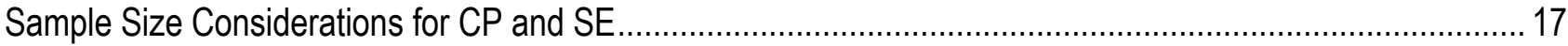

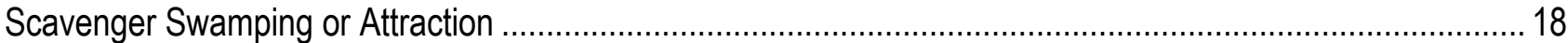

Placement of CP and SE Trial Carcasses for All Features ................................................................. 18

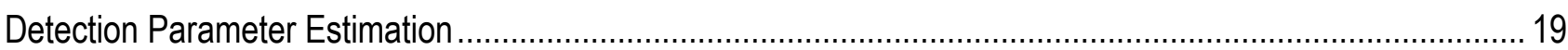

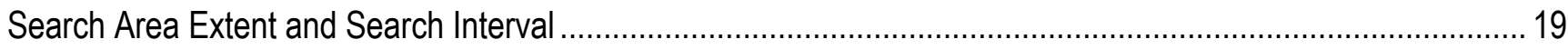

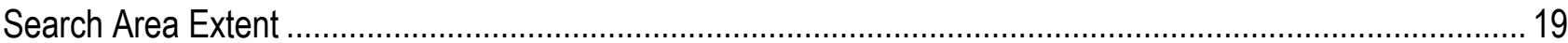

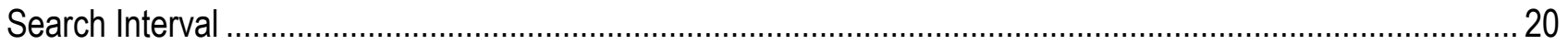




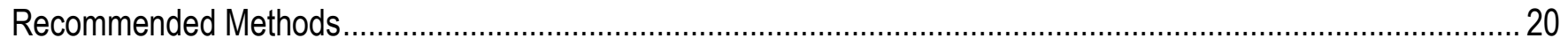

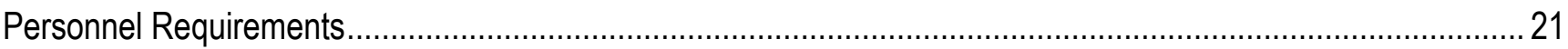

Data Collected for Each Carcass Observed................................................................................................ 21

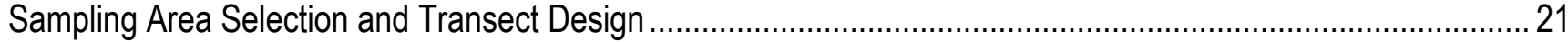

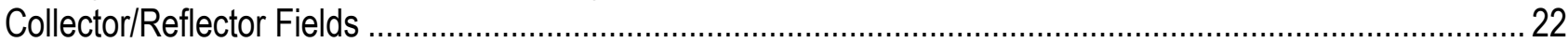

Power Tower (PT) within Central Circle............................................................................................ 22

Power Tower (PT) Beyond Central Circle ............................................................................................. 23

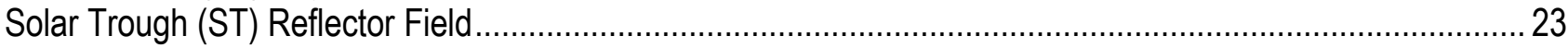

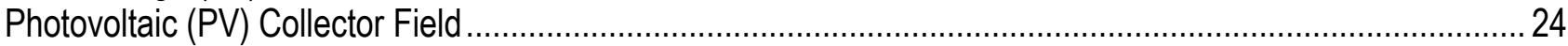

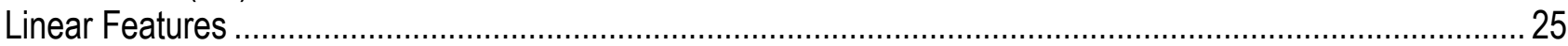

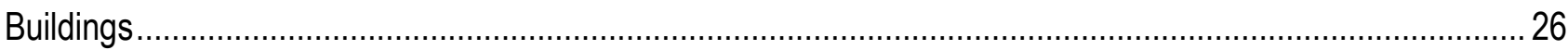

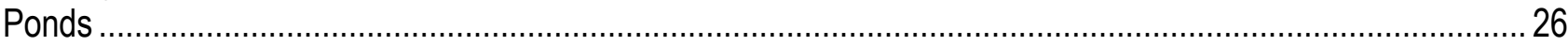

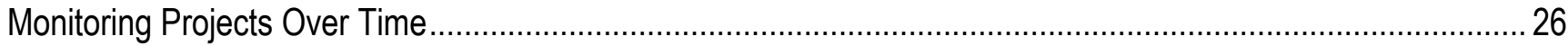

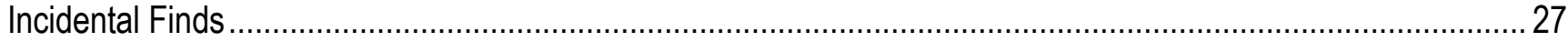

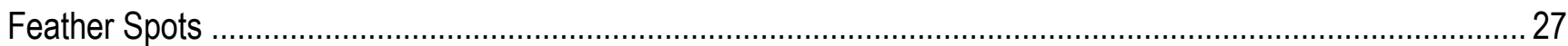

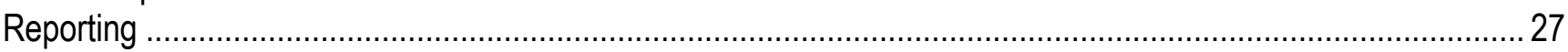

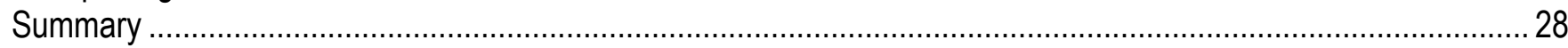

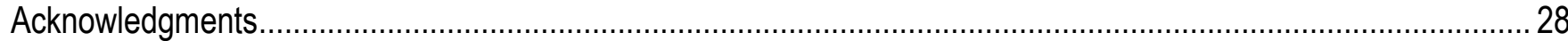

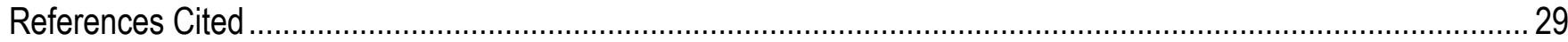

Appendix A. General Concept of Distance Sampling................................................................................ 32

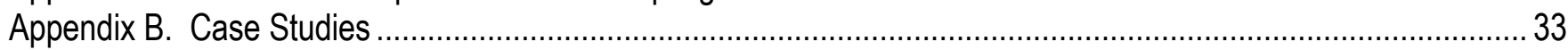

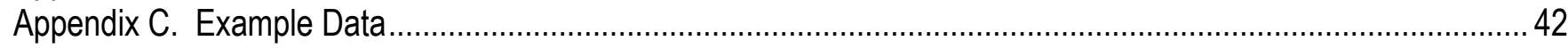

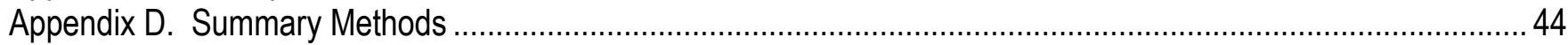

\section{Figures}

Figure 1. Schematic representation of monitoring design components ........................................................... 2

Figure 2. Photovoltaic collector field at Desert Sunlight Solar Farm showing "lake effect".................................... 8

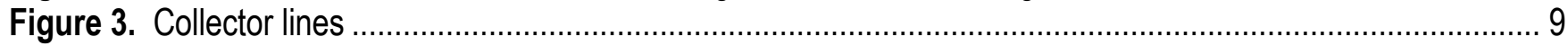

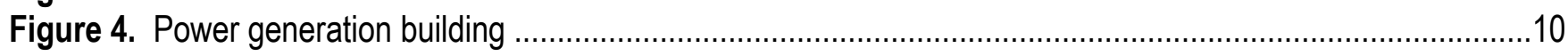

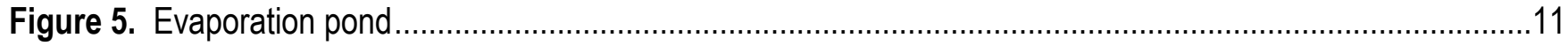

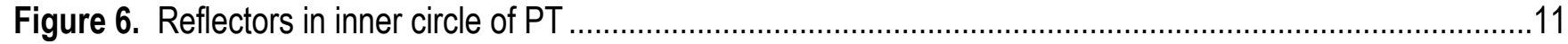

Figure 7. Desert scrub surrounding Desert Sunlight Solar Farm..................................................................12

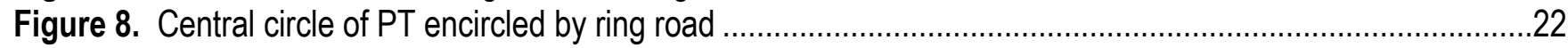

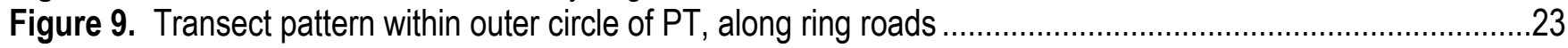

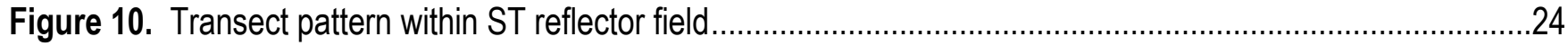

Figure 11. Transect pattern within photovoltaic arrays panel array ................................................................24

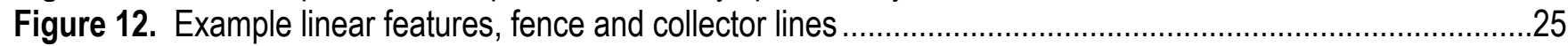

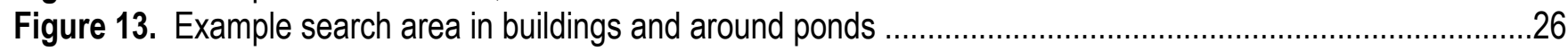

\section{Table}

Table 1. Project features on different solar technology types that represent different sources of mortality ….......... 7 


\section{Conversion Factors}

SI to Inch/Pound

\begin{tabular}{lccc}
\hline & Multiply & By & To obtain \\
\hline \multicolumn{4}{c}{ Length } \\
\hline meter & 39.3701 & inch & \\
kilometer & 0.6213 & mile & \\
\hline
\end{tabular}

Temperature in degrees Celsius $\left({ }^{\circ} \mathrm{C}\right)$ may be converted to degrees Fahrenheit $\left({ }^{\circ} \mathrm{F}\right)$ as follows:

$$
{ }^{\circ} \mathrm{F}=\left(1.8 \times{ }^{\circ} \mathrm{C}\right)+32
$$

\section{Datums}

Vertical coordinate information is referenced to the North American Vertical Datum of 1988 (NAVD 88).

Horizontal coordinate information is referenced to the North American Datum of 1983 (NAD 83).

Altitude, as used in this report, refers to distance above the vertical datum. 
This page left intentionally blank 


\title{
Mortality Monitoring Design for Utility-Scale Solar Power Facilities
}

\author{
By Manuela Huso ${ }^{1}$, Thomas Dietsch ${ }^{2}$, and Chris Nicolai ${ }^{2}$
}

\section{Introduction}

Solar power represents an important and rapidly expanding component of the renewable energy portfolio of the United States (Lovich and Ennen, 2011; Hernandez and others, 2014). Understanding the impacts of renewable energy development on wildlife is a priority for the U.S. Fish and Wildlife Service (FWS) in compliance with Department of Interior Order No. 3285 (U.S. Department of the Interior, 2009) to "develop best management practices for renewable energy and transmission projects on the public lands to ensure the most environmentally responsible development and delivery of renewable energy." Recent studies examining effects of renewable energy development on mortality of migratory birds have primarily focused on wind energy (California Energy Commission and California Department of Fish and Game, 2007), and in 2012 the FWS published guidance for addressing wildlife conservation concerns at all stages of land-based wind energy development (U.S. Fish and Wildlife Service, 2012). As yet, no similar guidelines exist for solar development, and no published studies have directly addressed the methodology needed to accurately estimate mortality of birds and bats at solar facilities. In the absence of such guidelines, ad hoc methodologies applied to solar energy projects may lead to estimates of wildlife mortality rates that are insufficiently accurate and precise to meaningfully inform conversations regarding unintended consequences of this energy source and management decisions to mitigate impacts. Although significant advances in monitoring protocols for wind facilities have been made in recent years, there remains a need to provide consistent guidance and study design to quantify mortality of bats, and resident and migrating birds at solar power facilities (Walston and others, 2015).

In this document, we suggest methods for mortality monitoring at solar facilities that are based on current methods used at wind power facilities but adapted for the unique conditions encountered at solar facilities. In particular, unlike at wind-power facilities, the unimpeded access to almost all areas within the facilities, the typically flat terrain, and general absence of thick vegetation allow distancesampling techniques (Buckland and others, 2001, 2004) to be exploited to advantage at industrial solar sites (see appendix A). These protocols build on the work of Nicolai and others (2011), and as our understanding and techniques for monitoring improve, the methods may be further modified to incorporate improvements in the future. We present case studies based on monitoring methods currently implemented at different utility-scale solar facilities to illustrate how distance-sampling techniques may improve overall detectability without substantially increasing costs. Every facility is unique, and the protocols presented may be adapted based on specific monitoring objectives and conditions at each site.

\footnotetext{
${ }^{1}$ U.S. Geological Survey.

${ }^{2}$ U.S. Fish and Wildlife Service.
} 
We provide guidance for designing monitoring programs whose objective it is to estimate the total number of bird and bat fatalities occurring at a facility over an extended period of time. We address spatial variation in causes of mortality, as well as potential sources of imperfect detection, for example, animals falling in or moving to unsearched areas, carcasses removed by predators, and carcasses missed by searchers. We suggest methods to estimate and account for each source of imperfect detection. This document focuses on monitoring design only and does not discuss approaches for estimating mortality from collected data. The development of statistically sound estimators relevant to the solar context is a current topic of research, although there are already strong foundations for estimation with distancesampling methods in similar open, arid environments (Anderson and others, 2001; Freilich and others, 2005). Nonetheless, if protocols described in this document are followed, the resulting data will be adequate and sufficient for estimating mortality using newly formulated estimators.

\section{Background}

Optimal monitoring design depends on the objective of the study and may vary among facilities. In carcass monitoring, the basic approach is to count carcasses observed in scheduled searches and divide by the estimated detection probability to give an estimate of the number of fatalities. The level of certainty associated with the mortality estimate depends on the observed count, the estimated detection probability, and the precision with which that value is estimated. In general, increasing the detection probability also increases the precision of the estimate (Thompson, 2012).

Optimal monitoring protocols involve balancing the need for mortality estimates precise enough to inform management decisions with the cost of sampling to achieve the needed level of precision. Optimal monitoring design begins by determining the level of precision needed to meet the mortality monitoring objective and a best guess at expected mortality over the period of interest (fig. 1).

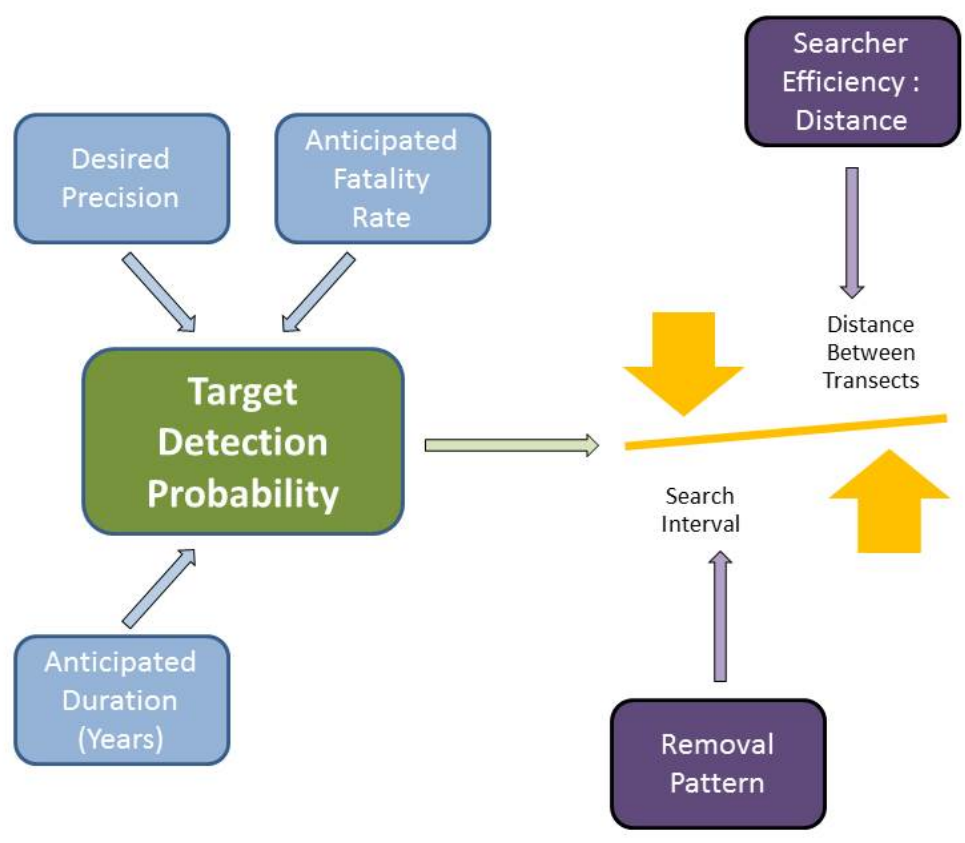

Figure 1. Schematic representation of monitoring design components. 
The overall detection probability for a species (or species group) will strongly affect the precision with which mortality can be estimated. The desired precision will differ depending on the objectives of the study (see section, "Goal and Objectives"). The target detection probability is defined as the overall probability of detection needed to be able to achieve the objectives of the study. The desired level of precision in the estimated mortality and expected mortality rate, for example, rare species with very low expected mortality versus common species with higher expected rate, are used to determine the target detection probability, which is also affected by duration of the study. Typically, the optimal interval between searches is determined by scavenging pattern, and the searcher efficiency for the site is a function of vegetation density and/or roughness of terrain as well as the distance between transects. The overall detection probability is reduced to a fraction when only a fraction of the total area is searched. Any number of potential combinations of time interval between searches, transect separation distance, and total area covered can result in the same target detection probability that are essentially equivalent from a statistical perspective, but may have very different implementation costs. A monitoring protocol that balances these three components while still achieving the target detection probability can be optimized to minimize costs. Repeated measures resulting from continuous monitoring throughout the life of the project can often be used to advantage to allow for a lower annual or seasonal target detection probability than when monitoring occurs for only 1 or 2 years (Dalthorp and Huso, 2015). The level of precision needed to achieve a general understanding of the order of magnitude of solar facility-caused mortality is usually far less than the level needed for testing hypotheses.

We address study design and suggest protocols for conducting monitoring studies to estimate the abundance of dead animals at each of three types of solar facility: power tower (PT), photovoltaic arrays (PV), and solar trough (ST), and in particular, for each potential source of mortality within a facility. When protocols for the different sources of mortality within facilities are similar, they are described under a single protocol. Data collected following these protocols should be adequate to achieve the stated objective of a monitoring program and inform adaptive management decisions.

We begin by discussing the two major components that comprise a monitoring program: (1) the protocol used to search for carcasses, which provides evidence of solar facility-caused mortality and addresses the goal of identifying species and taxonomic groups at risk; and (2) the protocol used to estimate detection probability of each carcass, with which observed carcass counts are adjusted to estimate total mortality. We discuss the potential sources of mortality at each facility type, and how search protocols and carcass detection probabilities might differ among the sources. For example, the persistence probability of a bird singed in the flux of a PT may be different than that of one killed by colliding with a reflecting mirror if singeing makes the bird more or less attractive to scavengers. We then discuss major causes of imperfect detection and how carcass persistence (CP) and searcher efficiency (SE) trials provide relevant data to detection probability models. We conclude with detailed recommendations on monitoring protocol for each facility type and each objective listed below. Our recommendations for monitoring protocol rely heavily on the use of distance-sampling techniques. Other methods (for example, simple random sample of a fixed fraction of equal area sampling units) are available and may provide equally relevant data, but for at least some situations, described in appendix B, Case Studies, we show how distance-sampling-based techniques can be equally as effective as, and less costly than, fixed area sampling. We recommend distance sampling techniques because of their flexibility in use across multiple species and because of their ease in implementation at solar facilities. 
We focus on appropriate monitoring and a field trial protocol that will provide data to accurately estimate the probability of detection of a carcass. This estimated probability as well as observed carcass counts are used to calculate estimates of mortality and associated uncertainty for the project area, for features within the project area or for specific periods of time within the overall study period. Estimates of mortality derived from collected data can be used to identify relative risk of different sources and/or can be combined to estimate total mortality at a site.

In this document, we focus on monitoring protocols that will yield observed carcasses with estimable probability of detection that can be combined using appropriate statistical tools to estimate mortality. A thorough presentation of the exact methods or algorithms for estimating mortality from solar facility monitoring data is beyond the scope of this document. Estimated mortality derived from data collected under the suggested protocols will represent fatalities within the confines of the facility and associated features only, hence will likely underestimate the total facility-caused mortality because fatally-injured animals dying outside the facility boundaries will not be accounted for. Some methods to account for carcasses beyond the searched area have been developed for wind-power facilities (Huso and Dalthorp, 2014), but this is an area of needed research for solar facilities.

\section{Goal and Objectives}

Our goal in developing this guidance is to assure that resulting mortality estimates will be comparable among sites, facility types, seasons, and years to inform wildlife management and natural resource policy decisions. The overall objective of this document is to provide guidance on how to conduct wildlife mortality monitoring to estimate the total number of bird and bat fatalities occurring at a solar facility. Objectives for mortality monitoring include: (1) estimating the total number and associated uncertainty of birds and bats killed at a facility within a specified time period; (2) determining whether there are spatial or temporal/seasonal patterns of mortality; (3) identifying species at risk; (4) evaluating changes in mortality due to adaptive management. Rigorous evaluation of some objectives should be addressed through formal hypothesis testing and may require modifications to monitoring protocol tailored to the specific conditions of the study site as well as the research question at hand. Exact study design for some research questions is beyond the scope of this document and should be developed in consultation with a trained statistician. However, a balanced application of this guidance can lead to helpful insights toward all stated objectives.

\section{Objective 1 - Monitoring to Estimate Total Bird and Bat Mortality}

The first objective of mortality monitoring at solar facilities is to provide a general understanding of the numbers of birds and bats killed at a facility over a defined period, usually a year or a season. Interpretation of the estimated number with respect to potential impacts will depend on the precision associated with the estimate, which in turn depends on the detection probability and precision with which it is estimated. 


\section{Objective 2 - Monitoring to Determine Spatial or Temporal/Seasonal Patterns of Total Bird Mortality}

Spatial Patterns.-Identifying spatial pattern associated with mortality within a project is important to (1) confirm assumptions regarding the distribution of carcasses; (2) provide information on avian behavior related to mortality; and (3) identify hazardous areas or components within a facility. The protocol recommended in this document relies on complete coverage of the facility and will provide data that can inform these questions.

Seasonal Patterns.- - Seasonal or other temporal patterns in mortality can provide insights into when birds and bats may be at greater risk. Identifying seasons with greater risk can inform timing of implementation of conservation measures. Known migration patterns or other annual life cycle information for species of interest can be used to define distinct seasons, although the monitoring protocol allows for seasons to be redefined if assumptions about migratory period are not correct or if additional information about the annual life cycle becomes available (for example, periods of juvenile dispersal or other regional movements). Regular searches at consistent search intervals and regular placement of SE and CP trials throughout the duration of the study, as recommended in this protocol, will provide data that can inform these questions.

Night versus Day.-Determining the time of day that fatalities are occurring may be useful for identifying appropriate measures to avoid or reduce mortality. The solar collector field may be perceived differently by passing birds at different times. Understanding when fatalities are occurring may be useful for engineers working to design project components with lower reflectivity or project configurations that break up visual aspects of a facility that may be attractive to birds. For some species, time of death (night versus day) can be inferred from knowledge of their behavior (for example, those known to be nocturnally or diurnally active), but for many species, behavior patterns may be unknown or may vary by activity (for example, foraging versus migration). For these species, addressing the hypothesis of greater (or lesser) mortality rate during night than day will require intense search effort (for example, twice daily searches at sunrise and sunset) and greater specificity of persistence rates. Duration of searches may need to be limited to only an hour or two immediately after dawn or before dusk in order to be relatively certain that the time of death of observed carcasses can be accurately assigned to the preceding night or day. Separate persistence trials for carcasses arriving during the night vs day may be necessary. However, studies whose objective is to test a hypothesis rather than to estimate an overall rate may be possible over a much smaller but representative sample area, which might help offset the cost of frequent searches and more persistence trials. Exact study design for research intending to test a hypothesis should be developed in consultation with a trained statistician.

\section{Objective 3 - Monitoring to Evaluate Which Species and Taxonomic Groups Are at Risk}

Species at risk.-A species' risk from solar facilities depends on multiple factors including abundance in the vicinity of the facility, individual bird movements near the project, and behavioral responses to the facility as they approach or interact with project components. Observed carcass data can inform potential risk, but imperfect detection likely prevents complete knowledge of the full list of species killed at a facility. However, relative risk may be inferred by comparing the estimated number of fatalities post-construction with pre-construction (or off-site) survey data, assuming appropriate trials to estimate detection probabilities are conducted independently in each area or time period. 
Taxonomic Groups/Behavioral Guilds.-For many species, the number of carcasses found may be too low to provide meaningful evaluation of relative risk. In this case, inference may be possible only for species combined into taxonomic groups or behavioral guilds - for example, passerines, waterbirds, or raptors. Relative abundance must be calculated from mortality estimates that account for differential detection rates among species and even individuals. Because detection rates are not constant, relative abundance based on simple counts will be biased.

Rare or listed species.-An "evidence of absence" approach similar to that developed for wind can be utilized to combine estimated detection probabilities with observed carcass counts (even when the count is 0 ) to estimate the likelihood that mortalities of rare species or species of special concern are below a set threshold (Dalthorp and others, 2014; Huso and others, 2014; Dalthorp and Huso, 2015). Target detection probabilities for rare or listed species will depend, among other things, on the threshold level (which may be the permitted take level set under an Incidental Take Permit), the desired level of assurance that number of fatalities of a species is below the threshold, and the duration and timing of the study (Huso and others, 2015). Dalthorp and Huso's (2015) comparison of outcomes under many different scenarios at wind facilities can inform choices at solar facilities as well.

\section{Objective 4 - Monitoring to Evaluate Changes in Mortality Due to Adaptive Management}

Meta-analysis. - Many questions arising from avian mortality issues at solar facilities may require compiling or comparing data across many projects. This document provides a protocol that allows for comparisons across different projects regardless of technology types and variations in specific methods (for example, search interval, transect separation distance, etc.)

Adaptive Management.-As monitoring results are generated, agencies and facility operators can work together to identify management practices that may reduce fatalities at a project. Adaptive management allows a framework for evaluating impacts and implementing conservation measures or making adjustments to management practices as needed (Williams and others, 2009; Williams and Brown, 2012). Monitoring over time is a critical component of adaptive management that allows facility operators and relevant agencies to evaluate whether changes in management practices are effective and whether additional or different measures are needed. This protocol is designed to collect data that can be compared across time or across facilities, although the ability to detect small, yet biologically significant effects will increase with increased detection probability. Exact study design for research intending to test the effectiveness of an adaptive management practice should be developed in consultation with a trained statistician.

\section{Sources of Mortality}

Solar technologies can differ greatly in the features they comprise, each of which represents a different source of potential mortality for birds and bats. Although some sources of mortality are common to all types of solar power facilities, for example, perimeter fencing or solar collector/reflector areas, other features are unique to certain types (table 1). At each site, the different sources of mortality may result in different detection probabilities, and optimal monitoring design may call for different search intervals, plot sizes, or plot selection processes. In addition, it is often of interest to estimate mortality rates at different features to determine where mitigation or avoidance efforts would have most impact. 
Table 1. Project features on different solar technology types that represent different sources of mortality.

[Features with similar monitoring protocol are grouped]

\begin{tabular}{llccc}
\hline \multicolumn{1}{c}{ Group } & \multicolumn{1}{c}{ Source of mortality } & $\begin{array}{c}\text { Power tower } \\
\text { (PT) }\end{array}$ & $\begin{array}{c}\text { Solar trough } \\
\text { (ST) }\end{array}$ & $\begin{array}{c}\text { Photovoltaic } \\
\text { (PV) array }\end{array}$ \\
\hline Collector/Reflector field & Solar collectors/reflector area & $\mathrm{X}$ & $\mathrm{X}$ & $\mathrm{X}$ \\
Linear features & Gen-ties & $\mathrm{X}$ & $\mathrm{X}$ & $\mathrm{X}$ \\
& Collector lines & $\mathrm{X}$ & $\mathrm{X}$ & $\mathrm{X}$ \\
& Perimeter and/or internal fence lines & $\mathrm{X}$ & $\mathrm{X}$ & $\mathrm{X}$ \\
Buildings & Operations management facilities & $\mathrm{X}$ & $\mathrm{X}$ & $\mathrm{X}$ \\
& Steam turbine facility & $\mathrm{X}$ & $\mathrm{X}$ & \\
Ponds & Evaporation ponds & $\mathrm{X}$ & $\mathrm{X}$ & \\
Power tower & Flux & $\mathrm{X}$ & & \\
Background & Background & $\mathrm{X}$ & $\mathrm{X}$ & $\mathrm{X}$ \\
\hline
\end{tabular}

\section{Attributing Mortality to Source}

If all fatalities were caused by collision impact with a structure, location of the carcass could provide information regarding cause, as these carcasses would likely fall relatively close to the point of impact, and signs of impact are sometimes evident as dust tracks, stains or feathers on collectors/reflectors or building walls. However, not all animals found close to a mortality source can be assumed to have been killed by that source. For example, stranded birds (unable to take off after landing) may seek shelter far from their initial point of arrival before succumbing to heat exhaustion, or a bird fatally injured when flying through the solar flux might end up anywhere throughout the facility and beyond. In such cases, attributing its death to the nearest source may not be justified. In addition, scavengers and wind can displace carcasses from the location of their demise. For each carcass found, field crews should record overt signs of injury (for example, singed feathers), the most likely source of mortality, and the level of confidence they place on their determination (for example, very-, moderately, not-confident). This information can be used to guide interpretation of data when identifying project components associated with highest mortality rates. 


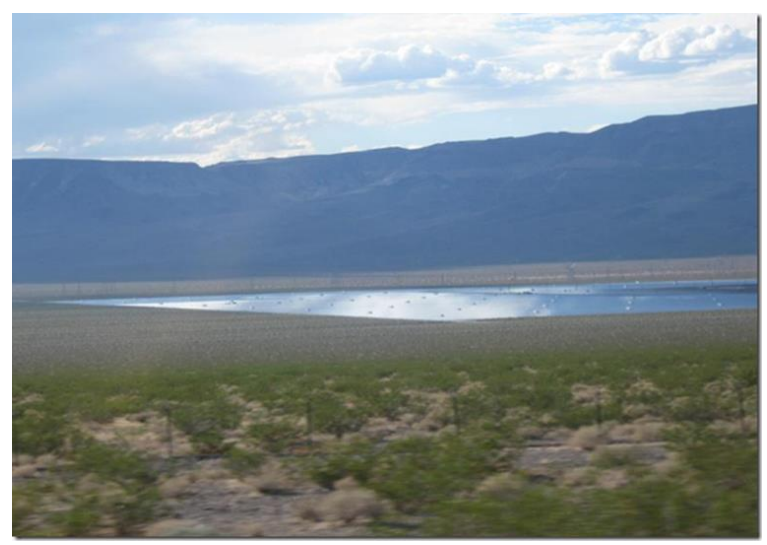

Figure 2. Photovoltaic collector field at Desert Sunlight Solar Farm showing "lake effect." (Photograph by Jody Fraser, U.S. Fish and Wildlife Service, 2015.)

\section{Collector/Reflector Field}

Solar collectors/reflectors cover the majority of the land area for utility-scale solar projects (fig. 1), creating opportunity for avian interactions and, thus, risk for birds. Birds may collide with collectors/reflectors that reflect the sky and clouds and are misconstrued as safe passage. Horvath and others (2009) hypothesized the "lake effect," where polarized light produced by PV solar facilities triggers maladaptive behaviors in birds and other wildlife, including attempting to land and forage on solar panels as though they were a body of water (fig. 2). Injuries from collisions with collectors/reflectors may result in immediate death due to fatal blunt trauma (Kagan and others, 2014), or stranding, that is, the inability of a bird to take flight. Stranding can occur when an individual crippled by collision impact is unable to take off, or when a water bird (for example, cormorant, loon, etc., that can reach take-off velocity only after running on the surface of a water body) lands safely but, without a sufficiently large body of water, cannot take off and may succumb to heat exhaustion. Stranded birds may not remain in the place where they initially land as they seek shade to avoid the heat.

Solar collectors/reflectors are typically raised off the ground, and vegetation below them is actively managed to varying degrees. High panels and cleared vegetation at some sites results in very high visibility across several independent structures. Carcass searches may be timed to coincide with periods when the position of movable solar collectors/reflectors allows for optimal visibility of the search area. 


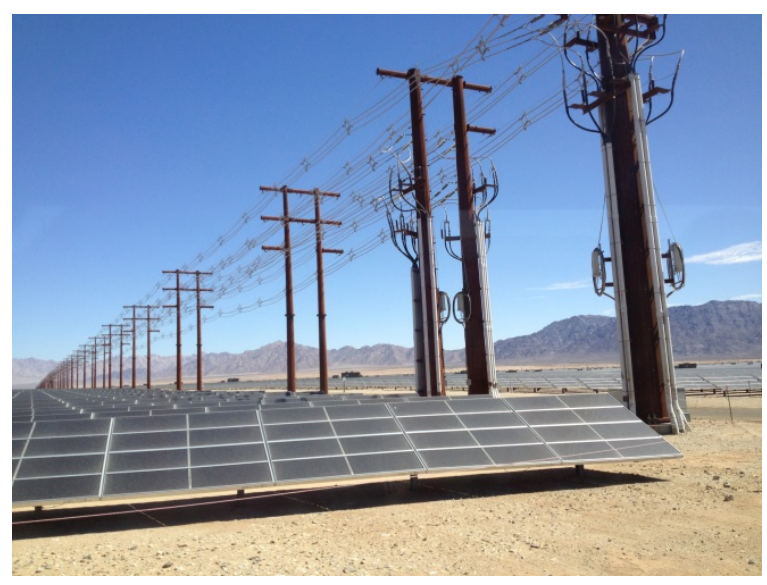

Figure 3. Collector lines.

\section{Linear Features}

Linear features such as generator-tie lines, collector lines, interior and perimeter fences, present collision hazard to birds, and electric lines represent a potential electrocution hazard (fig. 3). Similar to solar collectors/reflectors, mortality can be from fatal blunt force trauma or non-fatal injury that results in grounding. Injured birds may not remain on the premises but may move well beyond the limits of the facility before succumbing to their injuries. Electrocutions are often evidenced as scorching of the feathers, and carcasses are typically found fairly close to the electrocution site. Fence lines present a collision hazard to birds. However, not all carcasses found along fence lines can necessarily be attributed to this cause as birds injured by another source may accumulate along fence lines as they seek safety or shade. Feathers and other parts from scavenged birds may be moved by the wind and may also accumulate along fences. Consequently, fences represent an important feature to search, but some carcasses observed there may have been killed by other than interaction with the fence. 


\section{Buildings}

Collision is the major cause of bird mortality from buildings, but other causes include being caught in uncapped pipes, caught behind doors, and burned by super-heated parts in steam turbine facilities (fig. 4). Human activity is typically highest in these areas, increasing the chance of incidental discovery of a carcass. At some facilities, carcasses generated from collisions with buildings may overlap with those from other sources (for example, flux of PT). This may cause difficulty in correctly attributing cause but should not pose problems for correctly estimating total site mortality. Those areas that are safely accessible should be included in monitoring. Areas that cannot be safely accessed or that are out of view (for example, roof-tops that cannot be searched) should be accounted for in estimates of proportion of carcasses falling outside searched areas.

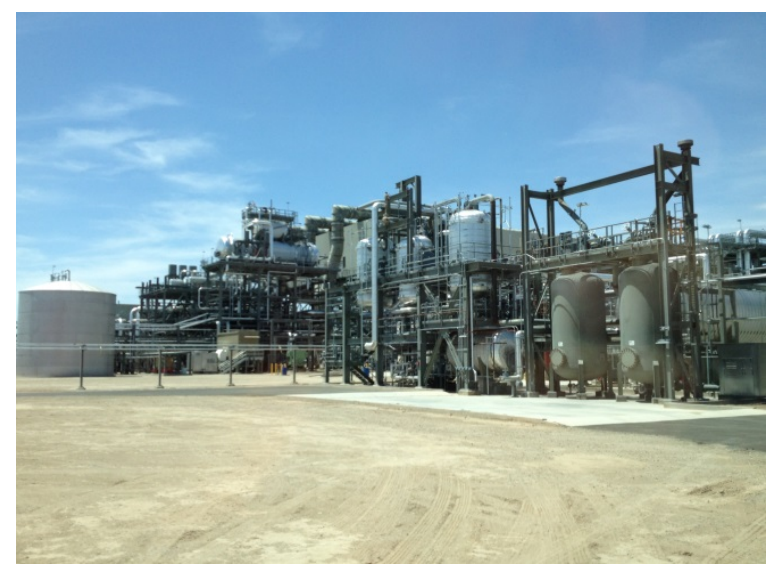

Figure 4. Power generation building.

\section{Ponds}

Open water, including evaporation ponds, attracts birds, especially in arid climates where solar energy facilities are frequently sited (fig. 5). Fatalities may occur due to water contamination, steep banks preventing escape from water, entanglements in netting, salt encrustation, etc. Netting exclosures on oil pits have proven successful in allowing efficient evaporation while keeping birds from entering the water and being exposed to concentrated salts and other toxic chemicals (Trail, 2006). However, netting may not be visible to birds from a distance and ponds may still be attractive to local and migrating water birds, placing them at risk from other sources of mortality within the facility. If a bird lands on the net, it can become entangled or unable to take off, effectively "stranded" and may eventually die from predation or heat exhaustion. Additionally, some birds may enter the exclosure through a small opening in the netting and become trapped inside, unable to find the same opening through which to exit, eventually dying from drinking contaminated water or heat exhaustion. 


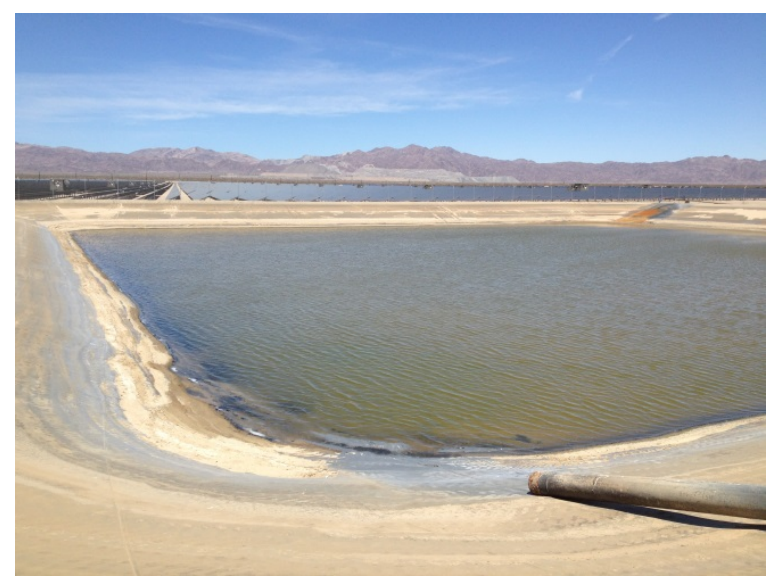

Figure 5. Evaporation pond.

\section{Power Tower}

Injuries from exposure to solar flux are an important and unique cause of mortality from power towers. These flux-related fatalities are more commonly found close to the tower (H.T. Harvey \& Associates, 2013). Birds may fly through the flux in the normal path of their flight, or may be attracted to areas of solar flux concentration while feeding on insects or other prey associated with the structure (fig. 6). Singed feathers on a carcass are evidence that the bird was killed by entering the flux (Kagan and others, 2014). However, not all birds entering the flux will exhibit singeing. Kagan and others (2014) hypothesized that some injured birds may wander within the facility or outside the project area, disoriented or unable to forage properly due to feather damage or blindness, before succumbing to exposure or predation. Again, different sources of mortality may be found within the same area, and while not affecting overall estimates of mortality, may make it difficult to correctly attribute mortality to its proper source.

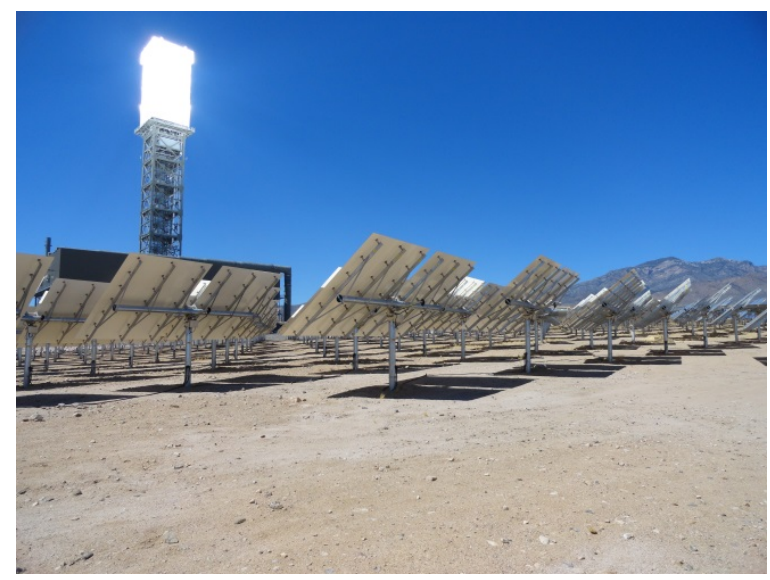

Figure 6. Reflectors in inner circle of PT. 


\section{Background Mortality}

Recently, questions have been raised about whether observed mortality rates and species composition on project sites are consistent with natural or background mortality rates and composition (fig. 7). Statistical methods to assess background mortality include conducting Before-After-ControlImpact studies (Hewitt and others, 2001) or paired or simple t-tests. Background mortality monitoring plots that are monitored while the solar plant is operating should be located far enough away to avoid the possibility that scavengers could transport carcasses to the background plots from the project infrastructure area, but close enough to assure that the background plot is representative of mortality in the area prior to construction. This distance should be greater than the home range size or local movement patterns of scavengers known to frequent the project area. The monitoring protocols outlined in this plan can be easily adapted to monitor background mortality, but specific recommendations are not provided.

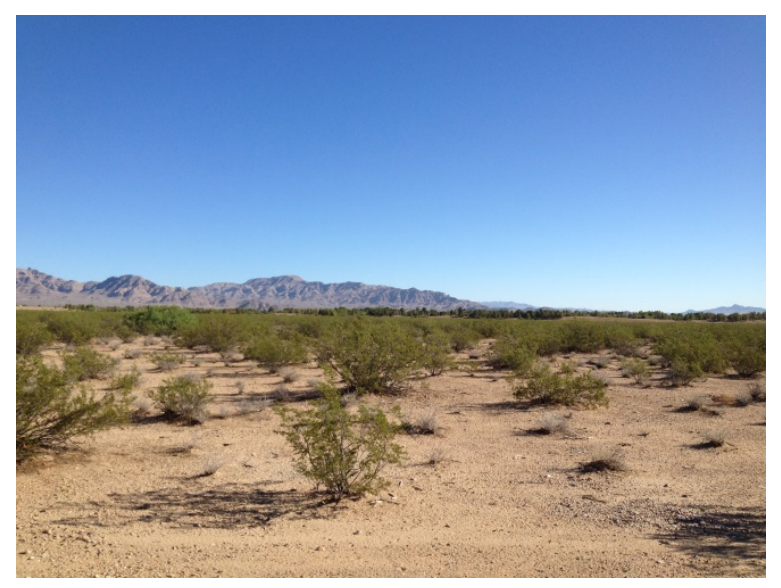

Figure 7. Desert scrub surrounding Desert Sunlight Solar Farm.

\section{Components of Monitoring}

Monitoring design involves two primary components: (1) searching for carcasses of animals killed at a solar facility, and (2) estimating the probability of detecting each carcass. Design elements of the search process primarily involve determining the location and lengths of search transects and the time interval between searches that are expected to result in the observation of a reasonable fraction of the carcass population at lowest cost. The two elements of the search process are not independent, and optimization of either will involve input from the other. Just as we must make assumptions regarding population variance when determining sample size in experimental design (Steele and others, 1997), we make assumptions regarding searcher efficiency functions and persistence rates in order to determine optimal transect length and location, and search intervals. The design elements of the search process are initially informed by assumptions regarding detection rates, but the actual probability of detection ultimately realized in the monitoring will need to be estimated. Thus, design elements of detectionprobability estimation involve determining numbers, characteristics, locations and timing of placement of trial carcasses used to determine detection probabilities of actual facility-caused fatalities during the monitoring period. Results from these trials, particularly if conducted prior to full monitoring, can inform initial decisions regarding the design elements of the search process, or may indicate useful changes to the original sampling design. 
An optimal search protocol (transect location and interval) is best determined by first identifying a target overall detection probability appropriate for the monitoring objectives. Among other things, detection probability can vary with size of an animal, where it is found, the season in which it is killed, and perhaps most importantly with the fraction of area of a solar facility in which searches are conducted. Carcass density for some mortality sources may not be constant, so search area and configuration relative to density play an important role in estimating mortality from observed carcasses (Huso and Dalthorp, 2014).

\section{Imperfect Detection}

The three primary reasons why not all animals killed at a solar facility are detected in the monitoring study are:

1. Some carcasses fall outside the sampled area: If a simple random sample of all equal area sampling units is feasible, the proportion of carcasses outside the sample can be represented as $(1-f)$ where $f$ is the sampling fraction. However, for some sources of mortality, a simple random sample may not be feasible, particularly when the area in which a carcass can land is not clearly defined; for example, the maximum distance at which animals striking transmission lines or fence lines may fall is difficult to determine, hence the total area in which a carcass could land is not clearly defined. In such cases, it may be necessary to develop models of carcass density as a function of distance from the feature to estimate the proportion falling beyond the limits of the searched area (for example, Huso and Dalthorp, 2014). With some sources of mortality in solar facilities we do not know if density changes with distance, but might be interested in determining if it does. In this case, it is important that the random selection of areas to search be constrained to be approximately equal at each distance from the source. This concept will be explained in more detail when we discuss sampling area selection.

2. Some carcasses are removed prior to a search: A fraction of the carcasses in an area is removed by scavengers, wind, or other means before the area is searched; therefore an estimate is needed of the proportion of carcasses expected to persist long enough to be detectable by a searcher, on the next and subsequent searches. In addition, a carcass missed on the first search following its arrival may persist until the next, affecting the overall SE. This "bleed-through" can be estimated by allowing searchers multiple opportunities to observe trial carcasses missed on previous searches.

3. Some carcasses are missed by searchers: Due to site conditions, proximity of the carcass to the observer, carcass morphology, searcher attentiveness and experience and pure chance, a fraction of the carcasses present during the search may be missed by a searcher, therefore an estimate is needed of the proportion of carcasses present at the time of search that a searcher is expected to find. This may be a decreasing function of distance from the searcher, in which case distance sampling techniques (Buckland and others, 2001) can be employed to determine the optimal transect width which results in an acceptable searcher efficiency. Efforts which can be taken to increase SE include use of dogs (H.T. Harvey \& Associates, 2014b) and truncation of transect width, although the former may preclude use of distance sampling techniques and the latter typically comes with a cost of decreased total searched area or increased costs. 
Each of these components represents a proportion (that is, a number between 0 and 1), and a very rough approximation $(\hat{g})$ of overall probability of detection $(g)$, although typically an underestimate, is to simply multiply them together, that is, $\hat{g}=\hat{a} \hat{r} \hat{p}$, where $\hat{a}$ is the estimated proportion of carcasses expected to fall in the searched area, $\hat{r}$ is the estimated proportion that remains within the area searched, and $\hat{p}$ is the estimated proportion of those that a searcher is expected to find. Thus, the same overall probability of detection can be arrived at from various combinations of $\hat{a}, \hat{r}$ and $\hat{p}$ but it can never be greater than the smallest component. For example, consider a site at which $60 \%$ of the small bird carcasses that arrive are expected to persist until the next weekly search. Searchers walking very narrow transects (for example, $10 \mathrm{~m}$ wide) over $50 \%$ of the site on a weekly basis could potentially observe $100 \%$ of carcasses that remain unscavenged (persist) within the search area. However, overall detection probability would be 0.30 because $100 \%(\hat{p})$ of the $60 \%$ that persist $(\hat{r})$ are observed in $50 \%$ of the total area $(\hat{a})$. Alternatively, searchers walking wide transects (for example, 50 m wide) over $100 \%$ of the site on a weekly basis might be expected to observe only $50 \%$ of the carcasses that remain unscavenged in the area. Again the overall detection probability would be 0.30 because $50 \%(\hat{p})$ of the $60 \%$ that persist $(\hat{r})$ are observed in $100 \%$ of the total area $(\hat{a})$. From a statistical perspective the outcome is essentially the same: searchers will observe approximately $30 \%$ of the carcasses, but from a cost perspective, the latter approach may be the more desirable as it achieves the target $g$ with only $40 \%$ of the search effort (see Case Studies for additional examples). An important consideration in developing the recommendations in this document is to maximize statistical power while reducing monitoring cost.

\section{Proportion of Carcasses in Sampled Area - $a$}

Extrapolation from a sample to the population depends on the representative nature of the sample. Typically, a random sample with equal probability of inclusion of each sampling unit assures representation (Cochran, 1977). If the rings of reflectors surrounding a central PT or blocks of collectors at a PV site could be subdivided into $N$ equal-area units from which $n$ were randomly selected to be searched, the sampling fraction $n / N$ would be a good estimate of $a$, the proportion of carcasses expected to be in the sampled area (for example, H.T. Harvey \& Associates, 2015). Alternatively, the entire area can be traversed and probability of observing a carcass modeled as a function of distance from the central transect line using distance sampling techniques (Buckland and others, 2004). With this approach, the sampling fraction is $100 \%$ and average searcher efficiency within the area is derived from the distance function (appendix A).

\section{Power Towers and Linear Features}

Variation in carcass density as a function of distance from the source, similar to what has been observed at individual wind turbines (Huso and Dalthorp, 2014), may be strongest in sources of mortality originating from a central location such as a power tower or a linear feature such as a transmission line. Density of carcasses killed by passing through the PT flux is expected to decrease with distance from the tower (perhaps non-linearly) both because fewer carcasses land at greater distances and because the area equidistant from a tower increases (Huso and Dalthorp, 2014). The distribution of carcasses generated by collisions with mirrors will overlap with the distribution of those caused by passage through flux, yet cause of mortality, other than singeing (for example, damaged retinas, dermis, or epidermis), may not be evident when examining a carcass. At PT facilities, the 
density of mirrors also decreases with increasing distance from the tower, and it is unknown whether carcass density is a function of mirror density or if fatalities caused by collision with mirrors will be evenly distributed throughout the mirror field. Because density of flux-caused mortality decreases with increasing distance from the tower and mirror-caused mortality may also decrease with decreasing mirror density, it is important that the sampling fraction be known at each distance from the tower. Constraining random selection of sampling areas to be a constant or known fraction at each distance class will assure that appropriate data will be collected to accurately assess the relationship of carcass density to distance from the tower and obviate the need to weight observations by sampling fraction. Alternatively, establishing transects centered on all ring roads (or half-way between ring roads; figs. 9 and 15) within a PT will increase coverage $(a)$ at a cost of decreasing SE $(p)$, thus realizing the same overall detection probability, but potentially at a lower or comparable monetary cost (see appendix B, Case Study 1, for example).

\section{Other Sources of Mortality}

Unlike PTs, density of solar collectors/reflectors at PV and ST facilities are typically uniform within blocks and sampling can be systematic. Although some injured animals may move from their original point of arrival, no available data indicate that the locations of the carcasses are inconsistent with an isotropic random process. At many facilities, visibility beneath collectors/reflectors is impeded only by structural supports; in others, unmanaged vegetation can obstruct view as well. Nonetheless, a systematic sample of widely spaced transects throughout the facility can yield an effective area coverage of near $100 \%$.

\section{Searcher Efficiency (SE) - $p$}

SE (the probability that a searcher will observe a carcass or feather spot present within the searched area) may vary with observers, ambient temperature, location within the facility, vegetation density, carcass or feather spot size, and particularly relevant to distance sampling, distance of the carcass from the observer. Solar facilities present an excellent opportunity for the application of distance sampling techniques (Buckland and others, 2001, 2004) because visibility is often good and the carcass population within a transect can be assumed to be approximately constant. When the density of the population whose abundance is being estimated can be assumed to be generated by an isotropic random process, distance sampling allows estimation of the average SE within the surveyed area. It is beyond the scope of this document to describe distance sampling techniques and methods for using sample data to estimate abundance, but when applicable, SE trial protocol recommendations are made based on the assumption that distance sampling techniques will be used (appendix A). SE and/or effective area surveyed should be estimated independently for each combination of factors that could lead to a substantially different level of SE. For example, SE under panels or mirrors where vegetation is beginning to grow back is likely to be lower than on tarmac surrounding Operations and Management (OM) buildings, and SE of small birds like wrens or warblers will likely be lower than SE of larger birds like pelicans or loons. SE models, whether distance-based or not, should account for these differences by incorporating relevant factors as covariates in SE models, and sampling protocol should assure that the covariate measures are recorded (see appendix $\mathrm{C}$ for example data sheets). 


\section{Carcass Persistence (CP) - $r$}

Different mortality sources within a facility may result in different persistence patterns, even for carcasses of the same size. For example, carcasses may persist longer near the OM buildings where there is a lot of human activity than in the mirror array because scavengers might avoid areas of high human activity. Or persistence times may be shorter because carcasses are more readily visible to scavengers willing to risk interaction with humans. Differential access to the project site or project features may also change the composition of the scavenger community and thus CP (for example, inside versus outside of fenced areas). Seasonal variation in the scavenger community is also an important consideration (for example, greater abundance of corvids during migratory periods).

Persistence trials are conducted to estimate proportion of carcasses expected to persist through search intervals of any length. Trials conducted prior to full-scale monitoring can inform study design and help determine staffing needs.

\section{Detection Trials and Potential SE and CP Covariates}

Several covariates that have been found to influence SE and CP rates at wind facilities (Gauthreaux, 1995; Morrison, 2002; H.T. Harvey \& Associates, 2014a, 2015) and are likely to apply to solar facilities include size of carcass, season, vegetation or visibility class, and individual searcher. The set of potential covariates may differ among the features/locations within a site, but at a minimum, size should be considered as a potential covariate for SE and season should also be considered as a potential covariate for CP. Typically, trial carcasses are placed in the field in adequate numbers to allow estimation of the effects of all potential covariates as well as their interactions with rates of SE and CP. Trial carcasses are placed regularly and discretely throughout each season, over a range of distances from the search transect line (appendix D) and are widely dispersed throughout the feature to best mimic the arrival process and to prevent either attracting scavengers or overwhelming them.

\section{Carcass Size}

Carcass size has been shown to influence both SE and CP-smaller carcasses are generally removed more quickly by scavengers and are less likely to be detected by a searcher than large carcasses (Osborn and others, 2000; Arnett and others, 2010). SE and CP should include size as a covariate, either as a class variable whose definition is determined in consideration of the species expected to be at risk at the solar facility, or as a continuous covariate. Because adequate numbers of local carcasses may not be available, it may be necessary to use surrogate species in detection trials. Surrogate species should resemble the local species at risk as closely as possible. Brown laboratory mice have been substituted for bats, starlings, and house sparrows for small birds; pigeons and quail for medium birds; and pheasants and ducks for large birds. Some studies have shown, however, that easily obtained surrogates may not accurately represent their intended group (Hale and Karsten, 2010; Niermann and others, 2011), so careful attention should be paid to selecting surrogates that not only mimic the size of the original species, but coloration and even flavor as perceived by scavengers. 
If vegetation is essentially homogeneous throughout a search area but is expected to change substantially among seasons, then season should be included as a potential covariate for SE. In this case, it may not be necessary to include vegetation/visibility class as a factor as this will be captured by the season covariate. If scavenging populations (for example, coyotes, ravens, ants, vultures) are expected to change substantially among seasons, then season should be included as a potential covariate for $\mathrm{CP}$.

\section{Vegetation/Substrate/Visibility Class}

If there are distinct types or densities of vegetation or substrate that are expected to affect a searcher's ability to see a carcass, then vegetation/substrate/visibility class should be included as a potential covariate for SE. If scavengers are expected to be more (or less) likely to forage when vegetation is high, then vegetation/visibility class should be included as a potential covariate for CP. As mentioned earlier, if vegetation is essentially homogeneous throughout a search area but is expected to change substantially among seasons, then season should be included as a potential covariate for SE, in which case it may not be necessary to include vegetation/visibility class as a factor.

\section{Individual Searcher}

Although searchers, regardless of training, will likely detect carcasses at somewhat different rates, adding individual searcher as an additional covariate is usually cost-prohibitive. In-depth training and pre-employment screening of those not meeting a minimum standard can help keep searcher efficiency rates approximately equal among observers. Placing SE trial carcasses randomly in the field, without attempting to balance exposure among searchers, but interspersed throughout the search schedule, will allow a natural weighting of the searchers' abilities and capture any improvement in searchers' skills over time.

\section{Sample Size Considerations for CP and SE}

In general, small carcasses are removed more rapidly and are more difficult for searchers to find than large carcasses, resulting in diminishing detection probabilities with decreasing size of the carcass. When detection probability is low, uncertainty in its estimate has a much stronger influence on precision of mortality estimates than does uncertainty when detection probability is high. We recommend that a minimum of 30 trial carcasses be used for bat and small bird trials for each combination of relevant covariates (for example, spring, central heliostat circle; or summer, fence line), whereas a minimum of 20 medium and 15 large bird carcasses is recommended to keep uncertainty in estimates to a reasonable level. These represent sample sizes for which the ratio of the upper: lower $95 \%$ confidence limits of SEs of $0.25,0.5$, and 0.75 is 6 -fold, 3 -fold, and 2-fold, respectively. They are arbitrary but attempt to balance statistical and economic costs. We emphasize that the recommended trial numbers are minimums for each combination of relevant factors and can and should be increased when feasible to increase precision of fatality estimates, particularly when SE is low. In addition, as there is some evidence (Gabe Reyes, H.T. Harvey \&Associates, written commun., February 2016) that feather spots are observed during searches with lower probability than intact carcasses, independent trials to estimate observerdetection functions separately for intact carcasses and feather spots should be considered. 


\section{Scavenger Swamping or Attraction}

Some authors have expressed concern that trial carcasses used to estimate detection factors may be changing the natural dynamics of a site (Smallwood, 2007; Smallwood and others, 2010, but see Huso and Erickson, 2013). In the previous section, we recommend that a total of at least 65 trial bird carcasses (split among three size classes) be placed randomly throughout a feature over a season. This represents less than one bird carcass per day above the rate generated at the feature and is unlikely to change the natural scavenging dynamics of a site. For features that cover a large area, for example, collector/reflector fields, this additional influx would be distributed over a very large area and is likely to have only trivial impact. For smaller features, for example, ponds or buildings, this additional influx may not be trivial and trial numbers can be reduced if necessary, particularly if $\mathrm{CP}$ and SE are relatively high.

\section{Placement of CP and SE Trial Carcasses for All Features}

For each feature group, SE trials should be conducted to provide estimates of the probability that a carcass within a searched area will be discovered by a searcher and CP trials will be conducted to provide estimates of probability that a carcass killed at a feature will persist between searches. With careful planning, the same trial carcasses can be used in both. Only carcasses that are fresh, that is, collected and frozen or placed in trial within 24 hours of death, should be used in CP trials. Trial carcasses should be placed at regularly spaced times throughout each season, not necessarily immediately preceding a scheduled search, and no more than two carcasses of each size class should be placed on a feature on any given day. This assures adequate sample size for estimating SE and CP, spreads out the carcasses' arrival times to keep scavengers from learning to anticipate the presence of carcasses, and captures any temporal variation there is in the scavenging process (see appendix B, Case Study 5).

Carcasses should be placed in the trial location by the crew leader without the knowledge of the search technicians. Often carcasses are tossed a short distance to mimic their landing at the site and to keep from leaving tracks or other signs that a trial carcass was placed. After placing a carcass, the crew leader should return to confirm the continued presence or the removal of the carcass daily for at least the first 4 days, then can increase the intervals between checks as the trial continues, if needed. An example schedule that fits well into the 5 day work-week schedule is to check on days $1,2,3,4,7,10,14,17,21$, 28 , or more often if opportunity arises. Monitoring carcass persistence frequently in the beginning and extending the monitoring well beyond the time of the average search interval will provide data needed to accurately estimate both parameters in two-parameter persistence models, appropriate when removal is not constant in time. Only under very unusual circumstances would a persistence trial study need to be extended beyond 28 days; for example, when scavengers are near absent for a season and carcasses persist for very long time, and hence the scheduled search interval is greater than 28 days. Alternatively, camera traps can be used to determine the exact date and time when a carcass is removed. Carcasses remaining on the final day of the trial can be removed from the area. CP carcasses should be inconspicuously marked to be able to distinguish trial carcasses moved by wind or scavengers from actual casualties. Carcasses should not be placed close to each other in either time or space (using the above methodology will assure this), reducing potential to attract scavengers above normal levels. If CP trial carcasses also serve as SE trial carcasses, the crew leader will check for the presence of a carcass not reported/recovered by the searcher as soon as possible after the searcher has left the area, to confirm 
its presence and that the searcher indeed missed it. It will then be left in place and its persistence monitored until it is either found, removed by a scavenger, or persisted the full 28 days. Leaving a carcass in place also allows a 2nd, 3rd, ... opportunity to be discovered after having been missed, thereby providing information needed to determine the parameter $k$, that represents the decrease in SE with repeated searches (Huso, 2011; Warren-Hicks and others, 2013; Wolpert, 2013; Huso and others, in press).

\section{Detection Parameter Estimation}

The general methods to calculate detection probability from data collected following the suggested methods given in this document are described in Huso and others (2012), Dalthorp and others (2014), and Huso and Dalthorp (2014), although exact calculations for detection probability using distance sampling techniques in this context are still under development. SE trial results are modeled using logistic regression models including distance as a continuous covariate and $\mathrm{CP}$ results are modeled using survival analysis methods (Lawless, 1982; Bispo and others, 2013). The covariates most important to SE and CP can be determined by AICc model selection (Burnham and Anderson, 2002; Huso and others, 2012).

As the desired precision of mortality estimates increases, the probability of detection necessary to achieve it will generally increase. A higher detection probability is usually achieved through more intensive monitoring, for example, more frequent or extensive searches, but can also be achieved by increasing a searcher's ability to find a carcass through the use of dogs, for example, or by increasing the persistence probability of a carcass by limiting scavenging.

\section{Search Area Extent and Search Interval}

The three primary causes of imperfect detection (that is, carcasses are outside the searched area, removed by scavengers or missed by searchers) interact to form an overall probability of detection. Higher detection rates relative to one cause can be traded off against lower rates from another cause to achieve a target overall detection rate. The two causes over which we generally have the most control are the areal extent of the survey and the frequency with which we search. The optimal search area, configuration and extent will depend on the technology employed at the facility. The optimal interval will be determined by the scavenging pressure, which may differ with location within the facility, season and size of carcass, among other things. A monitoring design appropriate for the species group most difficult to detect, will generally be adequate for all other species.

\section{Search Area Extent}

Because industrial solar facilities are established in generally flat, arid areas, the search area extent can be the entire facility, with each source of mortality sampled in its entirety, or it can be a random selection of equal area subplots covering a fixed proportion of the total area. Generally, searches will occur along fixed line transects. Optimal location and configuration of transects within the area depends on the visibility beneath collectors and reflectors and beneath linear features. Some facilities, for example, ST and some PV, actively clear vegetation, leaving an unobstructed view of the terrain except for the posts supporting the collectors or reflectors. Where vegetation is not cleared or is minimally managed, for example, some PV and PT beyond the inner circle, obstruction will result in a 
much more rapid attenuation of searcher efficiency with distance of the carcass from the observer (see

appendix B, Case Study 1, for example). Suggested transect designs will differ among facility types and among infrastructure groupings. Initial transect widths that coincide with natural pathways within the facility are logistically most efficient. If distance sampling assessment of SE indicates that searcher efficiency rates are higher (or lower) than needed to reach target detection goals, transect widths can be widened (or narrowed).

\section{Search Interval}

Prior knowledge of carcass persistence rates (see below) can be used to determine the optimal search interval. A short search interval is necessary only when average persistence time is also short. Consequently, we suggest that estimation of $\mathrm{CP}$ for each relevant class combination (for example, carcass size, season, vegetation class, etc.) be initiated prior to initiation of carcass searches. Because removal rates can often change with time, that is, age of carcass, estimating CP involves determining the best model, usually a two-parameter Weibull, log-logistic or lognormal model for removal time that allows rates to change with time since death rather than an exponential model that assumes constant removal rate. We recommend that the interval between searches be set by the class combination with the most rapid removal rate. An optimal search interval is set to complement the average searcher efficiency in such a way as to achieve the target overall detection probability. An interval that allows greater than or equal to $50 \%$ of the carcasses to persist to the next search can be used as an initial target, but can be modified once SE and effective area surveyed are calculated. Using a shorter interval will typically result in more precise estimates of mortality because a higher proportion of carcasses will remain to be found.

How well the observed carcasses represent the underlying fatality process throughout the study period depends on the search process. Weather, migratory patterns, and human activity patterns can all affect mortality patterns. Conducting searches on a regular basis will assure that temporal variation in the arrival (of carcasses to the site) process will be captured. For example, if optimal search interval is determined to be 10 days, then rotating through $10 \%$ of scheduled searches each day until all are completed will assure representation of the fatality process throughout the period, whereas conducting all searches on one out of every 10 days leaves any mortality estimator vulnerable to bias: If unusually large fatality events occur immediately following or immediately preceding the day of the search, subsequent estimates of mortality would be biased low or high, respectively.

\section{Recommended Methods}

In this section, we provide detailed guidance on recommended protocols for searching for carcasses and for designing carcass persistence and searcher efficiency trials at a solar facility for which no persistence or SE trials have previously been conducted (also see appendix D). Justification for our recommendations is described in earlier sections and in appendix B, Case Studies. Once SE and CP trials have been conducted and trial results evaluated, these recommendations can be modified to meet the specific conditions of each site and the objectives of each study. 


\section{Personnel Requirements}

Effective monitoring begins with the proper baseline skills and training of personnel as a prerequisite for collecting good data. The monitoring program should be adequately staffed, with work carried out by an independent crew leader with one or more technicians conducting searches. The crew leader should be a trained biologist or ecologist, capable of identifying the species of observed fatalities and collecting associated data. With a background designing and implementing similar studies, the crew leader can adapt to circumstances that arise during the study while maintaining scientific rigor. The crew leader should be responsible for conducting SE and CP trials, assuring that searchers are not aware of when they are being tested during SE trials once full monitoring commences. Technicians on the project do not need to be trained biologists, but should be trained in search protocol and tested prior to being formally added to the team. Those who consistently score low in searcher efficiency should receive additional training or be replaced with more vigilant searchers. The number of personnel required will depend on the size of the project, the effective area surveyed and the optimal search interval.

\section{Data Collected for Each Carcass Observed}

For transect-based searches outlined below, searchers should maintain a walking (or driving) speed as determined by the project manager and by the specific conditions in an area. There is no need to maintain the same speed in all areas; however, it is critical that the search process (including speed of searchers) be the same for trial carcasses as for actual fatalities. Immediately upon observing a carcass, the searcher should record the distance from the point of first observation to the carcass using a rangefinder. S/he should then continue searching along the transect line until s/he is at a point the shortest distance from the carcass, yet still on the transect line. The distance to the carcass should again be measured and noted as the perpendicular distance to the carcass (both these measurements can contribute to estimation of the observer-detection function.) The searcher will then leave the transect line, record relevant information (appendix $\mathrm{C}$, table $\mathrm{C} 1$ ), photograph and collect the carcass (if permitted), return to the transect line and continue searching. Alternatively, perhaps to take advantage of searching in cooler hours, after measuring both distances and photographing the carcass, the searcher can simply place a protective cover (for example, upended bucket with heavy weight to resist wind) over the carcass and return later to collect relevant information and retrieve the carcass (if permitted). State and Federal permits are required to handle carcasses of species protected under the Migratory Bird Treaty Act and Endangered Species Act. Carcasses for these species should be handled as directed by the appropriate agency, including carcasses found incidentally outside of the study area.

\section{Sampling Area Selection and Transect Design}

Location and extent of search transects will differ among the different solar facilities and features. The majority of transects will occur within the collector/reflector fields and along linear features. To optimize overall detection probability for all species, we recommend that searches be conducted along relatively wide transects that cover the full extent of each feature rather than covering only a randomly selected set of sample plots with relatively narrow transects within the feature. The advantage to full-extent transects is that the overall detection probability is maximized, that is, not limited by a sampling fraction less than one. Some facilities or areas within facilities are actively cleared 
of vegetation, leaving an unobstructed view of the terrain except for the posts supporting the collectors or reflectors, and allowing for wider transect spacing. Where vegetation is not cleared or is minimally managed, obstruction will result in a more rapid attenuation of the observer-detection function with distance of the carcass from the observer, and perhaps require narrower transect spacing and slower gait.

\section{Collector/Reflector Fields}

We recommend that the entire collector/reflector area of a facility be traversed by searchers moving along major and/or minor access paths whose exact configuration will differ by facility type. This effectively assures $100 \%$ coverage with the average probability of observing a carcass between transects determined by the observer-detection function based on distance sampling models (Buckland and others, 2001; see appendix A), and overall detection further determined by carcass persistence patterns and time between searches.

\section{Power Tower (PT) within Central Circle}

For Power Towers, the central reflector field typically extends about $275 \mathrm{~m}$ from the central tower (fig. 8) and is encircled by a ring road. The highest density of singed animals is likely to occur within this about 24 ha area and for many study objectives, it is often desirable to set search interval and transect widths to achieve a relatively high average SE. We recommend initial transect widths of 20-25 $\mathrm{m}$ following the natural rows between panels (appendix D, table D1).
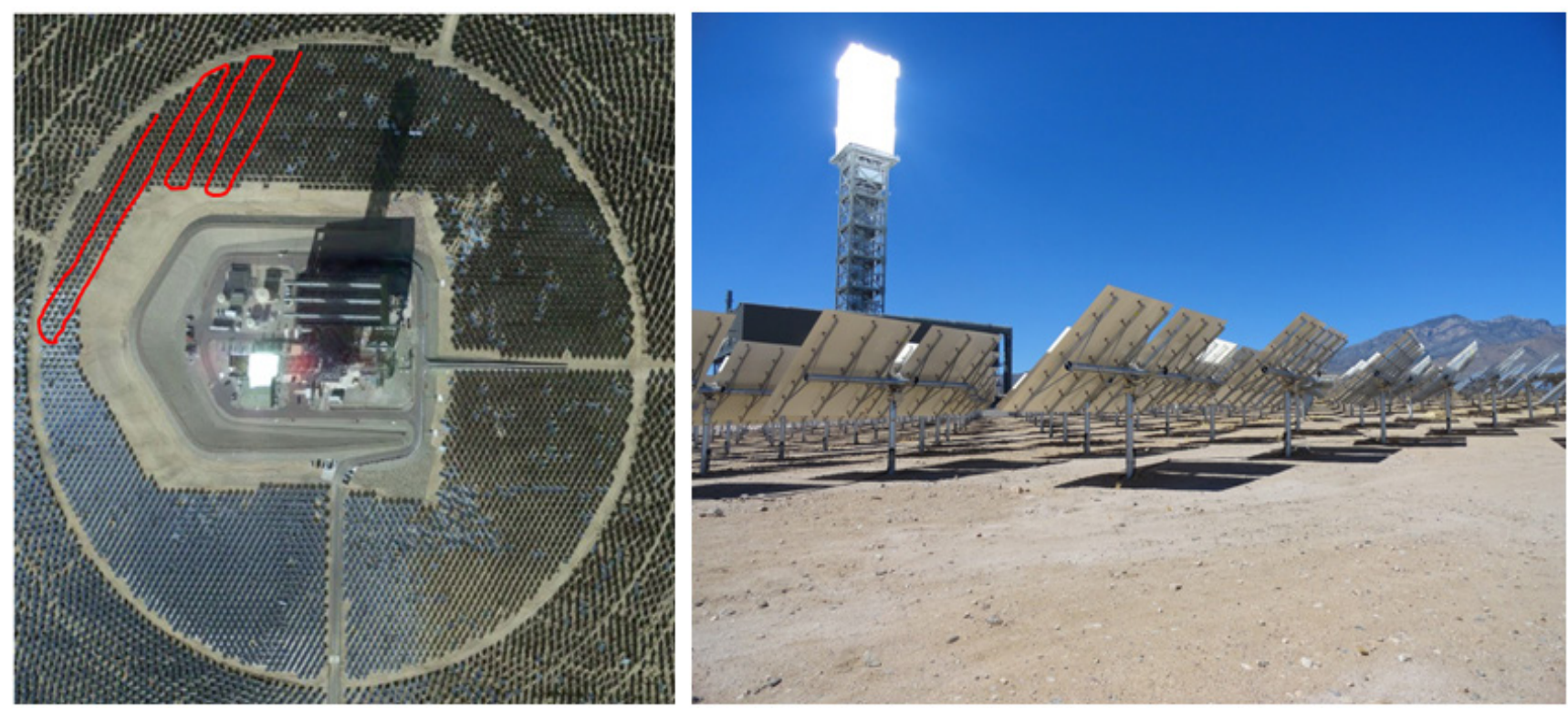

Figure 8. Central circle of PT encircled by ring road. PT and operations facilities are in the center. Meandering line in left image represents potential $25 \mathrm{~m}$ wide transect search pattern. 


\section{Power Tower (PT) Beyond Central Circle}

Ring roads occur at regular intervals from the central tower field and extend up to $1,500 \mathrm{~m}$ beyond the power tower. Radiating access roads form natural demarcations to divide the area into sections, each resembling a pie slice. For each section, a searcher walks along the ring roads in the pattern described in figure 9. The angle of the mirrors, positioned to reflect sunlight towards the central tower, may result in greater visibility towards the central tower than away (fig. 9). If so, field trials can determine if SE is higher when the searcher looks only to one side toward the central tower or to both sides (appendix A).
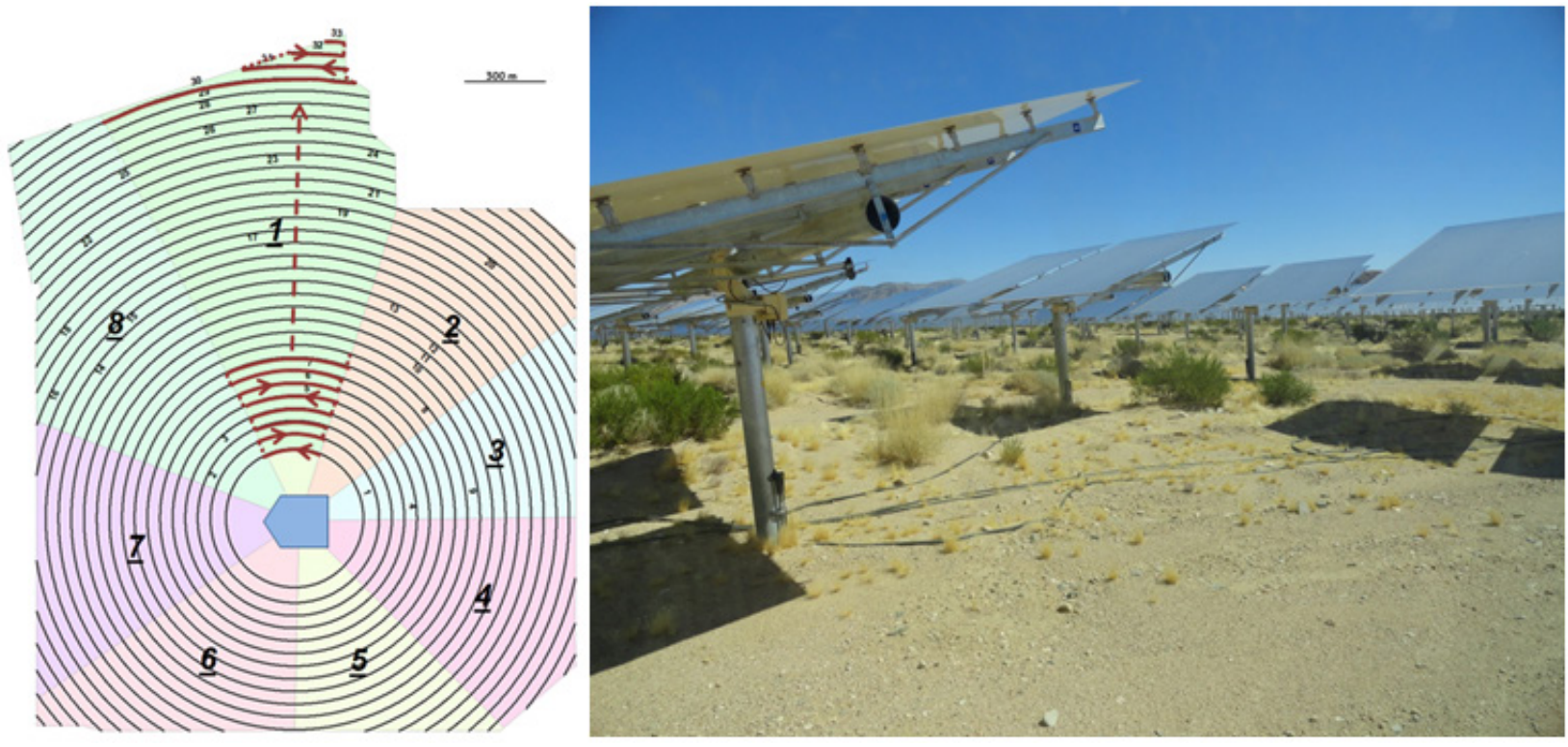

Figure 9. Transect pattern within outer circle of PT, along ring roads, that is, roads laid out in concentric circle surrounding the central PT.

\section{Solar Trough (ST) Reflector Field}

As with PT reflector fields, we recommend widely spaced transects throughout the ST field (fig. 10). The generally unobstructed view will likely result in fairly high SE, even for small carcasses. Ease of access may allow more rapid traverse of the area, for example, searchers in trucks or ATVs, on relatively short intervals, for example, twice daily or every other day, to achieve the target overall probability of detection defined for the study (see appendix B, Case Study 2). 

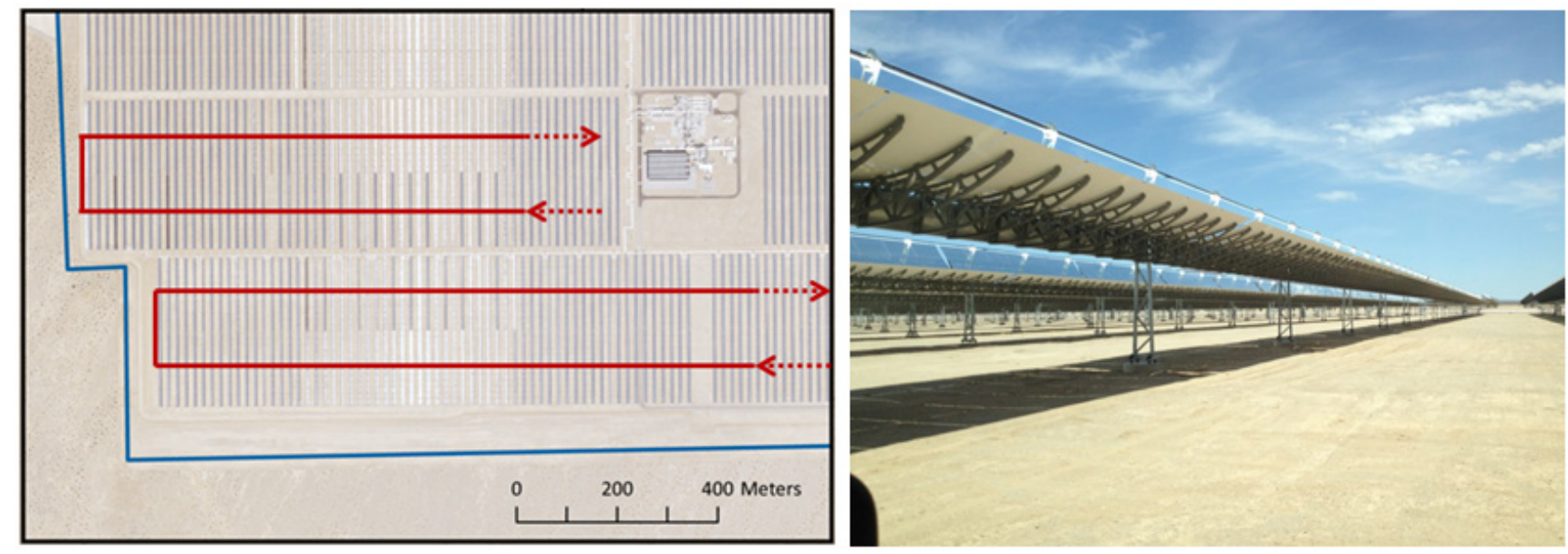

Figure 10. Transect pattern within ST reflector field.

\section{Photovoltaic (PV) Collector Field}

We recommend widely spaced transects along access roads, perpendicular to the panel rows (Case Study 3). Searchers walk slowly searching ahead and to both sides (fig. 11). Binoculars can be used to verify the presence of a carcass. Although edges of reflectors are often less than $2 \mathrm{~m}$ above the ground, visibility along PV rows from access roads is typically high in PV fields, particularly when vegetation is cleared, allowing for fairly wide transect widths. However, vegetation control in PV fields varies greatly among facilities and the distance between transects may need to vary accordingly to achieve the same target detection probability. Different observer-detection functions should be fit in areas where vegetation is managed and where it is not. The interval between searches will be determined in response to the average SE between transect lines, with longer intervals justified when SE is high.
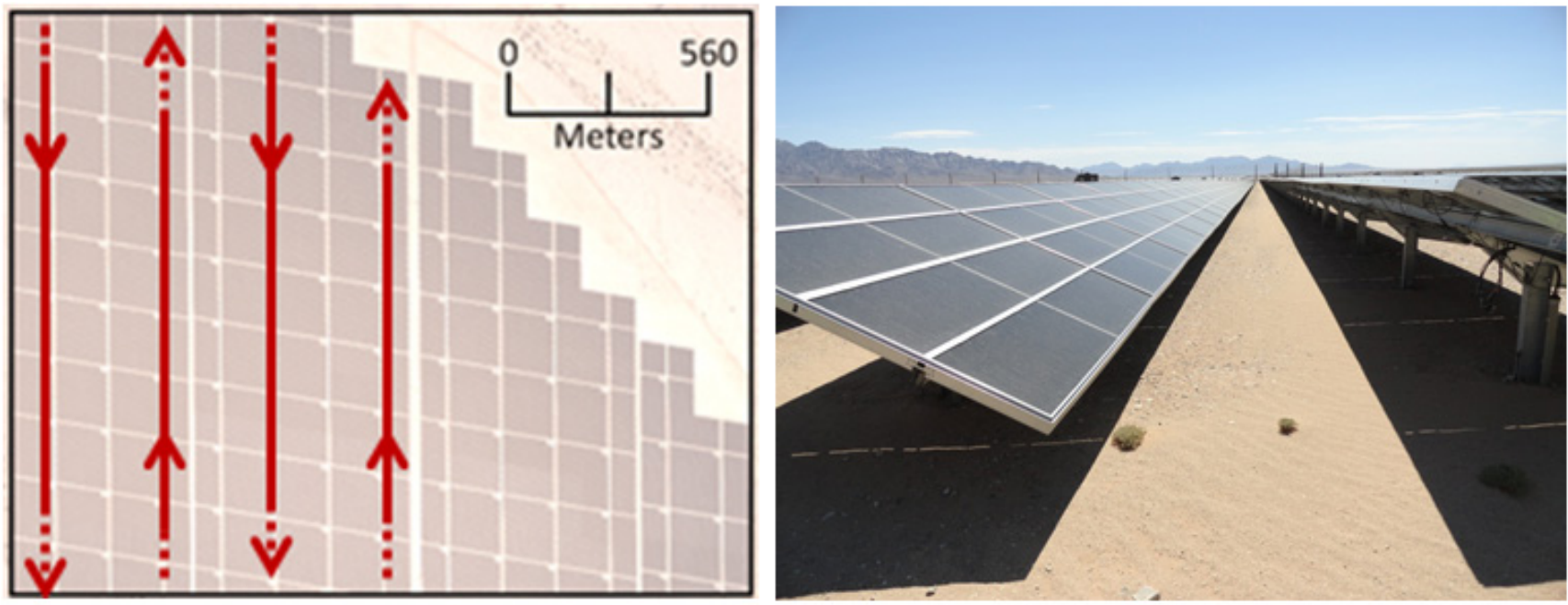

Figure 11. Transect pattern within photovoltaic arrays panel array. 


\section{Linear Features}

We recommend a full traverse of all linear features (fig. 12), where access allows, by searchers walking or driving in an ATV slowly along the fence line or under the power lines (Case Study 4). The overall detection probability will be reduced by the fraction of the linear feature that is accessible.
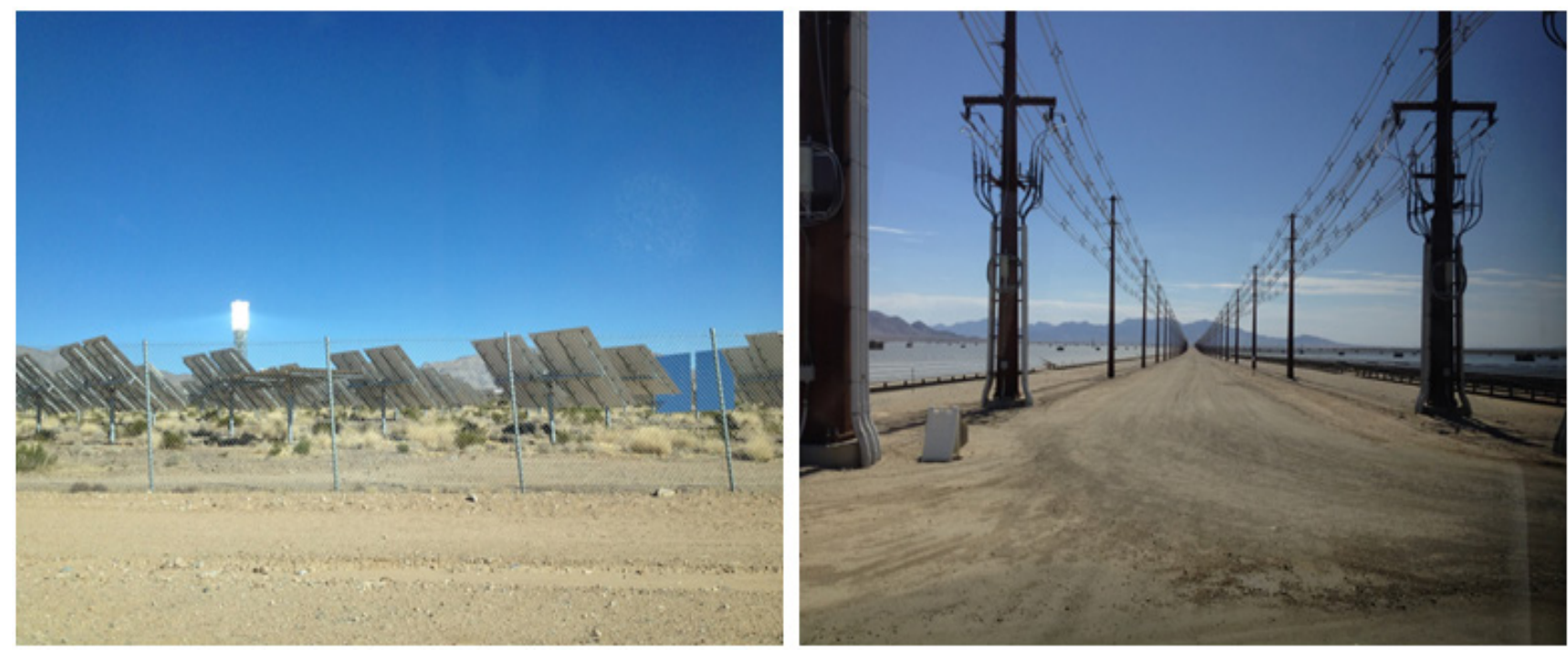

Figure 12. Example linear features, fence and collector lines.

Buildings (operations management centers and power generating structures) pose a unique situation regarding detection rates. Typically there is a lot of human activity in these areas and the opportunity for non-search personnel to find a carcass incidentally is high, yet excluding these carcasses from mortality estimation is problematic. We recommend that trial carcasses be placed in random locations within the area, and recorded as "removed" when the carcass is removed by a scavenger or found by a person, including non-search personnel (who should be trained to alert the crew leader when they discover a carcass, either trial or actual).

Scavenging rates and SE are combined into a single removal function. If trial carcasses are placed regularly throughout the study period, the removal process will be reflected in the data and will adequately reflect the same process that applies to a fatality. In addition to "incidental" finds, the search personnel will also perform regular searches. Because vegetation is typically cleared around buildings (fig. 13), they can generally be searched with $100 \%$ coverage by a searcher walking the entire area. Some buildings have walkways that can be followed, with searchers looking in all nooks and crannies that can safely be accessed. 


\section{Buildings}
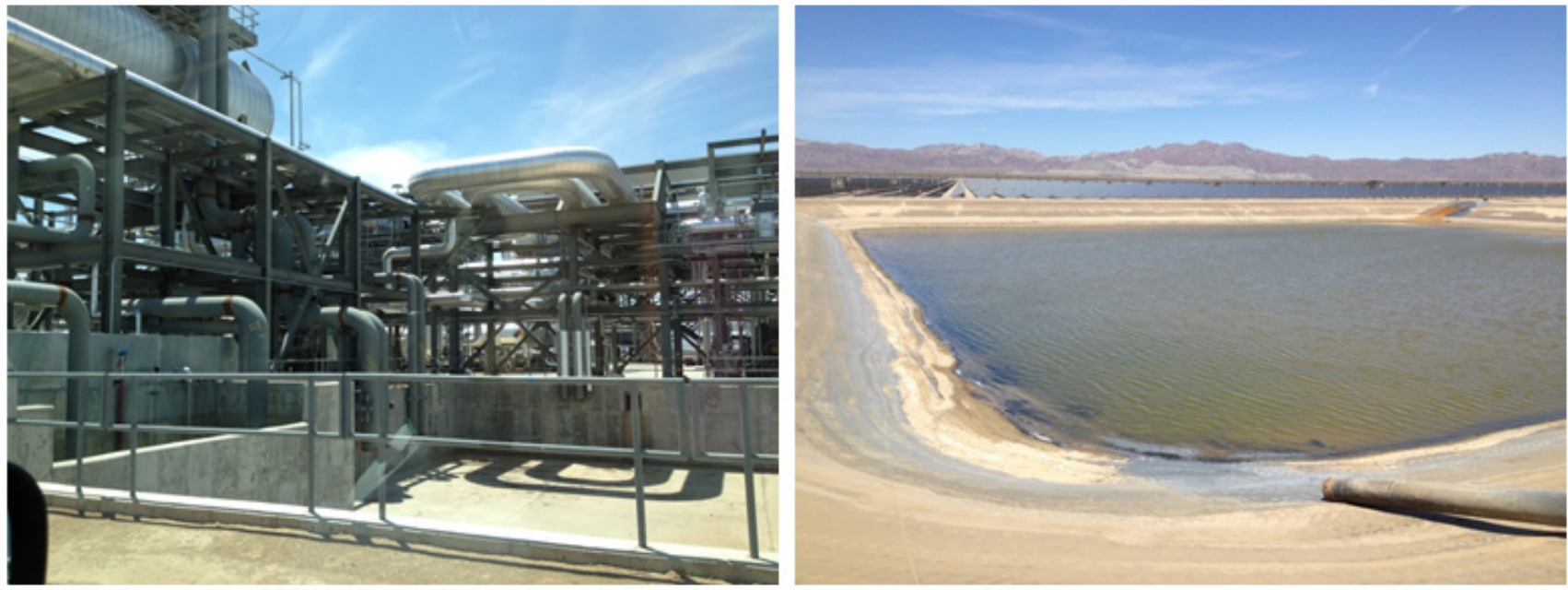

Figure 13. Example search area in buildings and around ponds.

\section{Ponds}

A searcher walking the entire perimeter of the pond (fig. 13) can achieve $100 \%$ coverage for this feature.

\section{Monitoring Projects Over Time}

Solar facilities are located in dynamic environments where macro-scale effects of climate patterns such as El Niño or La Niña, or micro-scale variation in rainfall pattern or even moon phase may affect the composition and magnitude of solar facility-caused mortality. Search intervals within a study period are typically very regular, for example, weekly, and only a fraction of the site is searched on any given day, for example, for searches conducted weekly on each weekday, one-fifth of the total area is searched daily. Any patterns of mortality due to micro-scale variation will be picked up in the search process. For macro-scale effects, the observed mortality in the first 1 or 2 years after operation begins may not necessarily be representative of mortality in years to come. Industrial-scale solar developments are a very new technology and we have little information regarding the variation in mortality pattern among facilities in different areas, among types of facilities and among years. To better understand how fatalities vary over time, mortality monitoring can be carried out continuously or with regular periodicity throughout the life of a project. The intensity of monitoring required throughout this period will depend on the specific objectives of the monitoring; achieving a rough understanding of the magnitude of the mortality impact will generally not require more than a fairly modest target detection probability whereas monitoring to assure compliance with an incidental take permit may require a higher continuous effort (Dalthorp and Huso, 2015). 


\section{Incidental Finds}

Some carcasses may be discovered by facility personnel or outside of scheduled search areas. Carcasses found incidentally near OM buildings or where human activity is concentrated, present a special case that we address above. In all other areas, carcasses that are found by non-search personnel but inside a monitored area should be documented and left in place to allow the monitoring process to play out, that is, discovery on a subsequent scheduled survey, removal by scavengers, or neither. If this is not possible due to agency requirements, these carcasses should be processed and removed as required, and carcass data collected for later use in mortality estimates, if appropriate.

\section{Feather Spots}

All available evidence of potential fatalities should be included in data used to estimate mortality rates at the facility. This includes degraded and dismembered carcasses that have been scavenged. Scavengers can be quite efficient in consuming a carcass, but can leave behind evidence that a fatality has occurred even if this is little more than a pile of feathers, referred to as feather spots (FS). The California Energy Commission and the California Department of Fish and Game (2007) have set standards for including FSs in carcass data at wind facilities. Searchers should make their best attempt to identify the species or taxonomic group of each carcass based on the sizes or identifying features of the feathers. There is some evidence (Gabe Reyes, H.T. Harvey \& Associates, written commun., February 2016) that FSs are observed during searches with lower probability than intact carcasses, particularly if the carcass and FSs are small. It may be necessary to estimate observer-detection functions separately for intact carcasses and FS.

\section{Reporting}

Regular reporting is important for keeping government agencies and other stakeholders informed of potential mortality issues at a project site. Quarterly reporting allows agencies an opportunity to provide feedback on the monitoring methods by reviewing results at regular intervals. If unusual species or unusually high numbers of fatalities are reported immediately, responsible authorities can take appropriate action in a timely manner. 


\section{Summary}

The recommended search protocols in this document rely heavily on the use of distance sampling techniques to maximize the coverage, that is, the extent of the area searched. Distance sampling is a viable sampling technique due to the unique characteristics of solar facilities relative to many wind facilities, that is, little if any impediment to access of all areas by searchers and sparse or cleared vegetation throughout the majority of the site. The use of distance sampling combined with the relatively high searcher efficiency on most projects allows for higher coverage than is currently achieved on many solar projects. As the case studies illustrate, 100\% coverage is often achievable with equal or less effort than is currently expended on many projects. However, distance sampling may not be optimal at heavily vegetated sites, and studies comparing distance sampling with conventional sampling under these conditions are needed. Although details of distance sampling techniques are beyond the scope of this document, following the recommended protocols will assure data collected will be appropriate for use in distance-based or more conventional estimates of SE.

In this report, we consider how imperfect detection influences design elements (coverage, searcher efficiency, and carcass persistence) for monitoring fatalities and discuss methods that can be applied for the different project features that cause fatalities at each solar technology type. For the major features of solar facilities (that is, solar collector field and transmission lines), trade-offs among the design elements can be utilized to optimize overall detectability. Although the distance sampling approach that we present should have broad applicability, we recognize that each facility will have unique characteristics and the study design may need to be adjusted as appropriate. Nonetheless, effective, well-designed mortality monitoring can assure that resources used to improve our understanding of the risks to wildlife from solar energy are wisely spent. The results will inform future efforts to avoid, minimize, and mitigate impacts from this valuable energy source.

\section{Acknowledgments}

We thank representatives at Desert Sunlight Solar Ranch, Ivanpah Solar Electric Generating System and Genesis Solar Energy Project for hosting our site visits to these facilities. We thank Dan Dalthorp for many fruitful discussions on distance sampling; Joel Pagel and Gabe Reyes for insightful comments generated from extensive field experience at solar facilities; Patti Haggerty for help preparing figures; Sarah Beldin for research support; and two anonymous reviewers, for comments and suggestions that served to improve this manuscript. 


\section{References Cited}

Anderson, D.R., Burnham, K.P., Lubow, B.C., Thomas, L., Corn, P.S., Medica, P.A., and Marlow, R.W., 2001, Field trials of line transect methods applied to estimation of desert tortoise abundance: Journal of Wildlife Management, v. 65, p. 583-597.

Arnett, E.B., Schirmacher, M.R., Hein, C.D., and Huso, M.M.P., 2010, Patterns of bird and bat fatality at the Locust Ridge II Wind Farm, Pennsylvania, 2009-2010 final report: Austin, Texas, Bat Conservation International, Prepared for the Bats and Wind Energy Cooperative and the Pennsylvania Game Commission.

Bispo, R., Bernardino, J., Marques, T., and Pestana, D., 2013, Modeling carcass removal time for avian mortality assessment in wind farms using survival analysis: Environmental and Ecological Statistics, v. 20, p. 147-165.

Buckland, S.T., Anderson, D.R., Burnham, K.P., Laake, J.L., Borchers, D.L., and Thomas, L., 2001, Introduction to distance sampling - Estimating abundance of biological populations: New York, Oxford University Press.

Buckland, S.T., Anderson, D.R., Burnham, K.P., Laake, J.L., Borchers, D.L., and Thomas, L., eds., 2004, Advanced distance sampling: New York, Oxford University Press.

Burnham, K.P., and Anderson, D.R., 2002, Model selection and inference-A practical informationtheoretic approach (2nd ed.): New York, Springer.

California Energy Commission and California Department of Fish and Game, 2007, California guidelines for reducing impacts to birds and bats from wind energy development: California Energy Commission, Renewables Committee, and Energy Facilities Siting Division, and California Department of Fish and Game, Resources Management and Policy Division, accessed CEC-7002007-008-CMF.

Cochran, W.G., 1977, Sampling techniques (3rd ed.): New York, Wiley \& Sons.

Dalthorp, D.H., and Huso, M.M.P., 2015, A framework for decision points to trigger adaptive management actions in long-term incidental take permits: U.S. Geological Survey Open-File Report 2015-1227. [Also available at http://dx.doi.org/10.3133/ofr20151227.]

Dalthorp, D.H., Huso, M.M.P., Dail, D., and Kenyon, J., 2014, Evidence of absence software user guide: U.S. Geological Survey Data Series 881, 34 p. [Also available at http://pubs.usgs.gov/ds/0881/.]

Erickson, W.P., Johnson, T., and Rabie, P., 2015, Post-construction monitoring at the Genesis Solar Energy Project, Riverside County, California: Juno Beach, Florida, WEST, Inc.

Freilich, J.E., Camp, R.J., Duda, J.J., and Karl, A.E., 2005, Problems with sampling desert tortoises-A simulation analysis based on field data: Journal of Wildlife Management, v. 69, p. 45-56.

Gauthreaux, S.A.J., 1995, Suggested practices for monitoring bird populations, movements and mortality in wind resource areas, in National avian-wind power planning meeting II, Palm Springs, California, September 20-22, Proceedings: Palm Springs, California, National Renewable Energy Lab, p. 88-110

H.T. Harvey \& Associates, 2013, Ivanpah solar electric generating system avian monitoring plan rev 12. California Energy Commission, Nipton, CA. Available from https://efiling.energy.ca.gov/Lists/DocketLog.aspx?docketnumber=07-AFC-05C.

H.T. Harvey \& Associates, 2014a, Avian and bat protection plan for the California Valley Solar Ranch Project: Santa Margarita, California, HPR II, LLC.

H.T. Harvey \& Associates, 2014b, Ivanpah solar electric generating system detection dog avian fatality trial: Nipton, California, Solar Partners I, II and VIII. 
H.T. Harvey \& Associates, 2015, Ivanpah solar electric generating system avian and bat monitoring plan, 2013-2014 annual report (revised): California Energy Commission, 67 p. https://efiling.energy.ca.gov/Lists/DocketLog.aspx?docketnumber=07-AFC-05C.

Hale, A., and Karsten, K., 2010, Estimating bird and bat mortality at a wind energy facility in northcentral Texas, in National Wind Coordinating Collaborative, Wind Wildlife Research Meeting VIII, Lakewood, Colorado, 2010, Proceedings: Lakewood, Colorado, National Wind Coordinating Collaborative, p. 13-17.

Hernandez, R.R., Easter, S.B., Murphy-Mariscal, M.L., Maestre, F.T., Tavassoli, M., Allen, E.B., Barrows, C.W., Belnap, J., Ochoa-Hueso, R., Ravi, S., and Allen, M.F., 2014, Environmental impacts of utility-scale solar energy: Renewable and Sustainable Energy Reviews, v. 29, p. 767-779.

Hewitt, J.E., Thrush, S.E., and Cummings, V.J., 2001, Assessing environmental impacts -Effects of spatial and temporal variability at likely impact scales: Ecological Applications, v. 11, p. 1502-1516. Horvath, G., Kriska, G., Malik, P., and Roberston, B., 2009, Polarized light pollution-A new kind of ecological photopollution: Frontiers in Ecology and the Environment, v. 7, p. 317-325.

Huso, M.M.P., 2011, An estimator of wildlife fatality from observed carcasses: Environmetrics, v. 22, p. 318-329.

Huso, M.M.P., Dalthorp, D., Dail, D., and Madsen, L., 2014, Estimating turbine-caused bird and bat fatality when zero carcasses are observed: Ecological Applications. DOI:10.1890/14-0764.1 Available from http://www.esajournals.org/doi/pdf/10.1890/14-0764.1.

Huso, M.M.P., Dalthorp, D., Dail, D., and Madsen, L., 2015, Estimating wind-turbine-caused bird and bat fatality when zero carcasses are observed: Ecological Applications, v. 25, p. 1213-1225.

Huso, M.M.P., Dalthorp, D., and Korner-Nievergelt, F., in press, Vulnerable species-Statistical principles of survey design and monitoring, in Perrow, M., ed., Wildlife and windfarms_Conflicts and Solutions, Volume 1-Onshore: London, Pelagic Publishing.

Huso, M.M.P., and Dalthorp, D.H., 2014, Accounting for unsearched areas in estimating wind turbinecaused fatality: Journal of Wildlife Management, v. 78, p. 347-358.

Huso, M.M.P., and Erickson, W.P., 2013, A comment on "novel scavenger removal trials increase wind turbine-caused avian fatality estimates": Journal of Wildlife Management, v. 77, p. 213-215.

Huso, M.M.P., Som, N., and Ladd, L., 2012, Fatality estimator user's guide: U.S. Geological Survey Data Series 729. [Also available at http://pubs.usgs.gov/ds/729/.]

Kagan, R.A., Viner, T.C., Trail, P.W., and Espinoza, E.O., 2014, Avian mortality at solar energy facilities in Southern California-A preliminary analysis: National Fish and Wildlife Forensics Laboratory. Available from http://alternativeenergy.procon.org/sourcefiles/avian-mortality-solarenergy-ivanpah-apr-2014.pdf.

Lawless, J., 1982, Statistical models and methods for lifetime data: Hoboken, New Jersey, John Wiley \& Sons, Inc.

Lovich, J.E., and Ennen, J.R., 2011, Wildlife conservation and solar energy development in the desert southwest, United States: Bioscience, v. 61, p. 982-992.

Morrison, M., 2002, Searcher bias and scavenging rates in bird/wind energy studies: Golden, Colorado, National Renewable Energy Lab, NREL/SR-500-30876, http://www.osti.gov/bridge.

Nicolai, C., Abele, S., Beeler, H., Doster, R., Kershner, E.L., and McCabe, T., 2011, Monitoring migratory bird take at solar power facilities-An experimental approach: U.S. Fish and Wildlife Service. 
Niermann, I., Brinkmann, R., Korner-Nievergelt, F., and Behr, O., 2011, Systematische schlagopfersuche-Methodische rahmenbedingungen, statistische analyseverfahren und ergebnisse, in Brinkmann, R., Behr, O., Niermann, I., and Reich, M., eds., Entwicklung von methoden zur untersuchung und reduktion des kollisionsrisikos von fledermäusen an onshore-windenergieanlagen: Göttingen, Cuvillier Verlag, p. 40-115.

Osborn, R.G., Higgins, K.F., Usgaard, R.E., Dieter, C.D., and Neiger, R.D., 2000, Bird mortality associated with wind turbines at the Buffalo Ridge Wind Resource Area, Minnesota: American Midland Naturalist, v. 143, p. 41-52.

Smallwood, K.S., 2007, Estimating wind turbine-caused bird mortality: Journal of Wildlife Management, v. 71, p. 2781-2791.

Smallwood, K.S., Bell, D.A., Snyder, S.A., and Didonato, J.E., 2010, Novel scavenger removal trials increase wind turbine-caused avian fatality estimates: Journal of Wildlife Management, v. 74, p. 1089-1097.

Steele, R.G.D., Torrie, J.H., and Dickie, D.A., 1997, Principles and procedures of statistics-A biometrical approach (3rd ed.): Boston, McGraw-Hill.

Thompson, S.K., 2012, Sampling (3rd ed.): New York, John Wiley \& Sons, Inc.

Trail, P.W., 2006, Avian mortality at oil pits in the United States-A review of the problem and efforts for its solution: Environmental Management, v. 38, p. 532-544.

U.S. Department of Interior, 2009, Order No. 3285-Renewable energy development by the Department of the Interior: U.S. Department of the Interior. [Also available at http://www.blm.gov/or/energy/opportunity/files/order_3285.pdf.]

U.S. Fish and Wildlife Service, 2012, U.S. Fish and Wildlife Service Land-based wind energy guidelines: Web page, accessed January 15, 2015 at http://www.fws.gov/windenergy/guidance.html.

Walston, Jr., L., Rollins, K.E., Smith, K.P., LaGory, K.E., Sinclair, K., Turchi, C., Wendelin, T., and Souder, H., 2015, A review of avian monitoring and mitigation information at existing utility-scale solar facilities: Argonne National Laboratory and National Renewable Energy Laboratory, ANL/EVS15/2, http://www.evs.anl.gov/downloads/ANL-EVS_15-2.pdf.

Warren-Hicks, W., Newman, J., Wolpert, R., Karas, B., and Tran, L., 2013, Improving methods for estimating fatality of birds and bats at wind energy facilities: Berkeley, California Wind Energy Association. (accessed CEC-500-2012-086)

Western Ecosystems Technology, Inc., 2015, Post-construction monitoring at the Desert Sunlight Solar Project: Cheyenne, Wyoming, Western Ecosystems Technology, Inc.

Williams, B.K., and Brown, E.D., 2012, Adaptive management: U.S. Department of the Interior Technical Guide.

Williams, B.K., Szaro, R.C., and Shapiro, C.D., 2009, Adaptive management-The U.S. Department of the Interior Technical Guide: U.S. Department of the Interior.

Wolpert, R., 2013, Appendix B-A partially periodic equation for estimating avian mortality rates, in Warren-Hicks, W., Newman, J., Wolpert, R., Karas, B., and Tran, L., eds., Improving methods for estimating fatality of birds and bats at wind Energy facilities: Berkeley, California Wind Energy Association, p. A1-A20. 


\section{Appendix A. General Concept of Distance Sampling}

Assume that observer-detection rate is a function of the distance from transect to carcass and that carcasses are randomly distributed throughout the search area. Let $f(x)$ be the probability of observing a carcass $x$ meters from the observer, where the exact shape of the function can depend on certain covariates discussed earlier, for example, size, season, associated feature, etc. (fig. A1). Then the average observer-detection rate of carcasses between two transects $w$ meters apart, when viewed from only one transect, is:

$$
\pi_{1}(w)=\frac{\int_{0}^{w} f(x) d x}{w}
$$

If a carcass that lies between two transects may be observed from either transect, then the average observer-detection rate of carcasses between two transects $w$ mapart is:

$$
\pi_{2}(w)=\frac{\int_{0}^{w}[f(x)+(1-f(x)) f(w-x)] d x}{w}
$$

The distance function can be estimated from $n$ trial carcasses placed at $0, \frac{w}{n}, \frac{2 w}{n}, \ldots \frac{(n-1) w}{n}$ meters from the transect, and well dispersed along all transects throughout the feature area, noting whether the carcass was observed during a search or not.

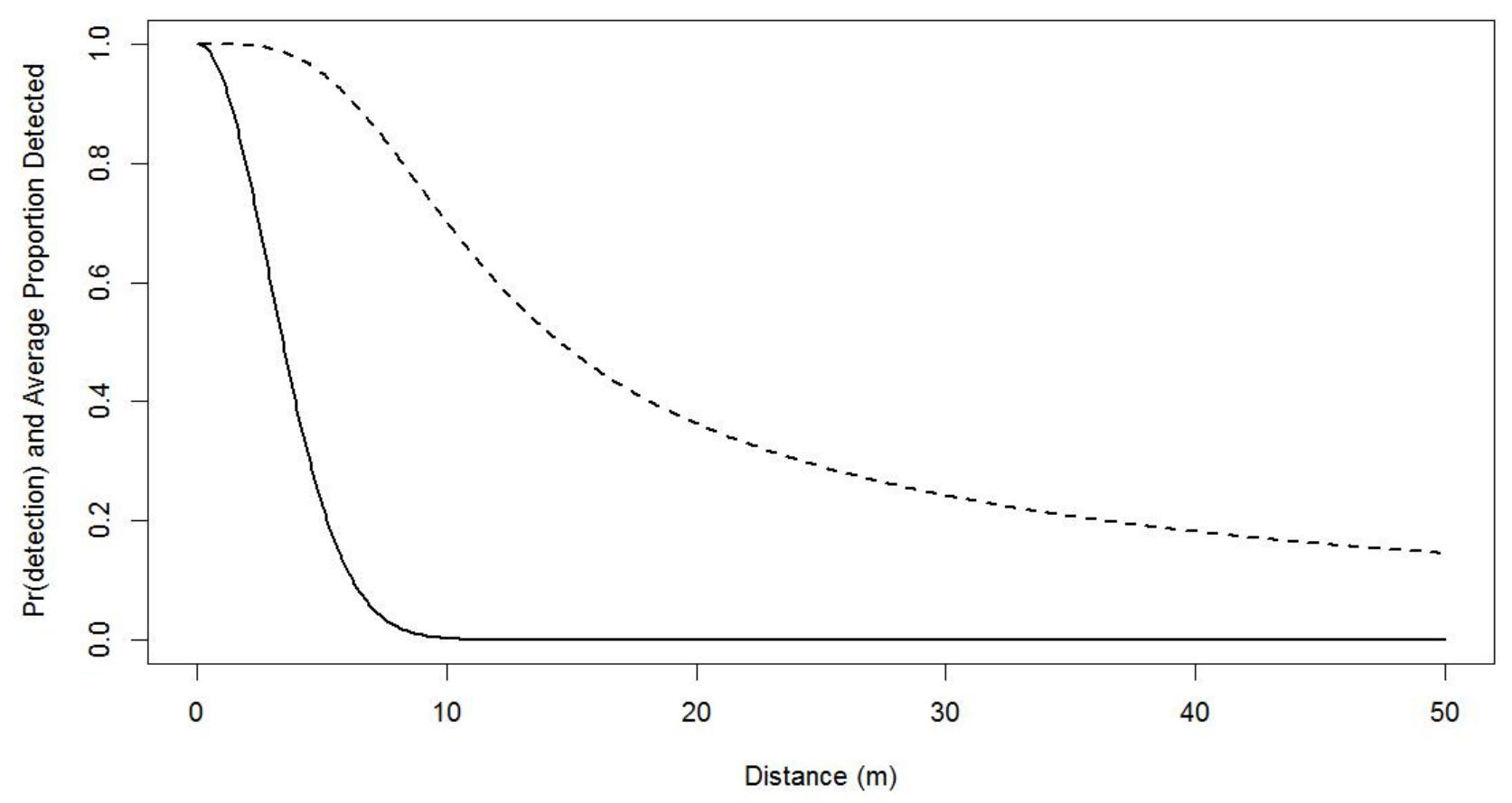

Figure A1. Example probability $f(x)$ of detecting a carcass $x \mathrm{~m}$ from the observer $(-)$, and average probability of $\pi_{2}(w)$ of detecting a carcass between two transects $x \mathrm{~m}$ apart (- - ). The function $f(x)$ represented here is a half-normal with standard deviation of 2.9 , and standardized to a value of 1 at $x=0$. 


\section{Appendix B. Case Studies}

Case Study 1 - 100\% Traverse of Circle Roads Versus 20\% Sample with 10 m Wide Meandering Transect

H.T. Harvey \&Associates (2015) proposed a sampling process in the heliostat field at Ivanpah, California as: "Twenty (20) percent of each heliostat field will be visually inspected for evidence of avian mortality or injury, according to the schedule in table 1. Sampling units will consist of 5-acre arc plots distributed throughout the heliostat fields, as shown in figure 2" (reproduced in fig. B1a). Each arc plot was traversed using 10-m-wide transects (fig. B1b) following a meandering path.
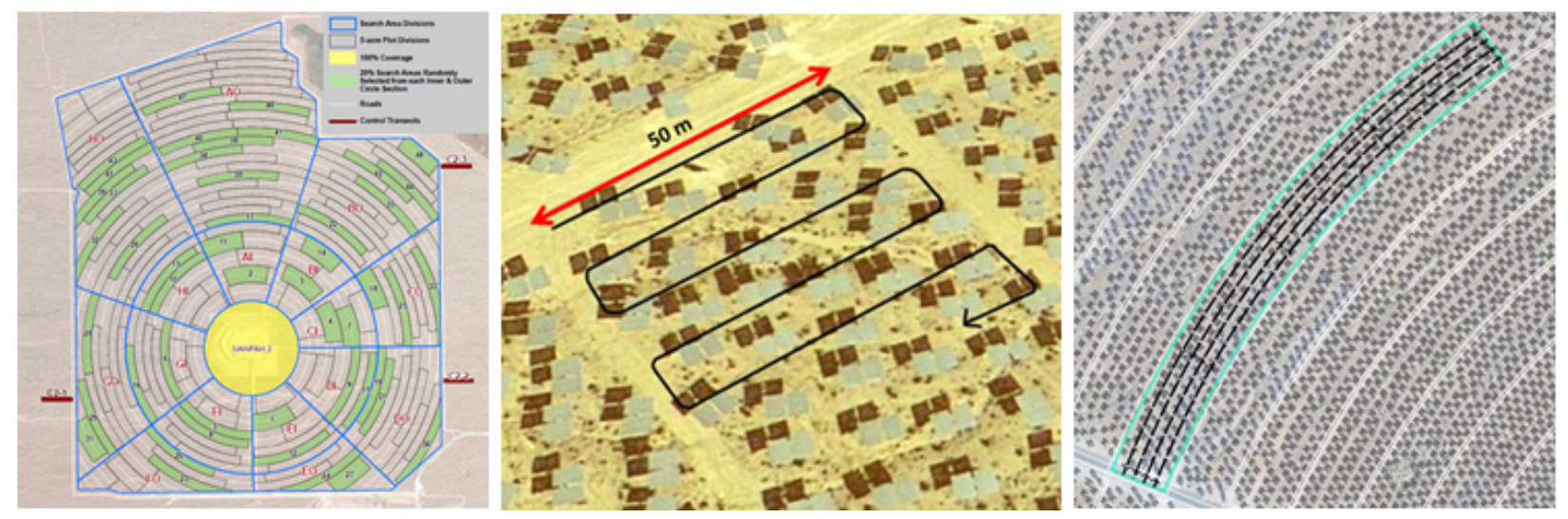

Figure B1. (a) Reproduction of figure 2 from H.T. Harvey \& Associates (2015) showing searched area representing $20 \%$ of total area beyond central ring of Tower 2 at Ivanpah; (b) Representation of initially proposed search path within a sample arc at Ivanpah; (C) Actual search path, summer 2014. Reproduced with permission from H.T. Harvey \& Associates (2015).

An alternative search pattern, utilizing a distance sampling approach, would be to traverse the entire heliostat field along the major circle roads. The total length of search path is approximately $20 \%$ greater following the proposed meandering path than it is walking/riding/driving all circle roads; however, our analysis suggests that the two paths can produce comparable overall detection rates ${ }^{1}$.

H.T. Harvey \& Associates estimated that $41.20 \%$ of small bird carcasses in the heliostat field would be detected by searchers (H.T. Harvey \& Associates, 2015). Searching only $20 \%$ of the heliostat field would result in overall detection probability of approximately $0.41 \cdot 0.2=0.08$. Although they do not fit an observer-detection function to their observations, a half-normal detection function, with standard deviation of 1.645 meters, results in an average SE of 0.412 within $10 \mathrm{~m}$ and gives a reasonable representation of an observer-detection function that could be associated with their data (see appendix A for calculations).

\footnotetext{
${ }^{1}$ Let $\mathrm{x}=$ circumference of one circle road. Then $0.2 \mathrm{x}$ is the length of the road that will be traversed by a searcher using the meandering transect approach. In addition to this length, each arc will have $0.2 \mathrm{x} / 10$ transects of $50 \mathrm{~m}$ each. The total search path then is $0.2 x+x$, hence $20 \%$ longer path than simply following the road.
} 
When using the road-based search protocol, the observer-detection function would extend out the full $50 \mathrm{~m}$ between ring roads in the heliostat field surrounding a PT, resulting in an estimated average detection of 0.08 over the entire field. This estimate is equivalent to the one based on the meandering search within $20 \%$ of the heliostat area, yet can be achieved through $20 \%$ less sampling effort $^{2}$. For larger birds, the estimated detection probability over the heliostat area was $0.14(0.68 \cdot 0.2)$ using the meandering approach, and only slightly better using the distance-based circle-road approach. However, under better visibility conditions, the combined detection rate for large birds has the possibility of rising above 0.20 , whereas with the meandering approach through a $20 \%$ sample, detection of any sized bird can never be greater than 0.20 . Although in the meandering approach observer-detection rates between transects will be higher because birds can be seen from either direction, this demonstrates a potentially high loss of efficiency in covering virtually the same area twice.

In summer 2014, the protocol was modified to run the transects $10 \mathrm{~m}$ apart, within the heliostat array and parallel to the circle roads, but still covering a $20 \%$ sample of the heliostat field (fig. B1c). Their estimated SE was higher than anticipated: $64.7 \%$ of small bird and $100 \%$ of large bird carcasses in the heliostat field sample plots were detected by searchers (H.T. Harvey \& Associates, 2015), resulting in 0.129 and 0.20 overall detection probability, respectively, over the site. Half-normal detection functions, with standard deviation of 3.46 and 11.2 (assuming 95\%, not 100\% detection within $10 \mathrm{~m}$ ), respectively, give reasonable representations of these data. Widening the transect to $50 \mathrm{~m}$ and traversing $80 \%$ of all ring roads represents an equivalent sampling effort relative to the implemented approach. However, facility-wide estimated average observer-detection probabilities of small and large birds are 0.138 and 0.51 , representing a roughly equivalent detection rate for small birds, but $>2$-fold increase in detection rate for large birds.

${ }^{2}$ Let $\mathrm{x}=$ circumference of one circle road. Then 4 parallel transects $10 \mathrm{~m}$ apart within each ring will result in $0.8 \mathrm{x}$ total transect length, or $20 \%$ shorter path than traversing all ring roads. 


\section{Case Study 2 - 100\% Traverse of Reflector Field Versus 30\% Sample}

Erickson and others (2015, section 3.7) reported SE of 0.92 (95\% CI: 0.85-1.00) for small birds within the searched area at the Genesis ST facility, Riverside County, California. This protocol was later changed, but serves a heuristic role in this discussion. Searches were conducted in sampling units covering $30.4 \%$ of the area beneath the solar troughs with an estimated observer-detection probability for the facility of about $0.28(=0.92 * 0.304)$. The sampled area was traversed using transects $300 \mathrm{~m}$ long separated by $30 \mathrm{~m}$, the width of two troughs: "Biologists slowly drove ( $\leq 5 \mathrm{mph}[8 \mathrm{kph}]$ ) parallel to troughs and centered between rows, searching ahead and to the driver's side of the vehicle for bird and bat carcasses. Biologists scanned out to a perpendicular distance of approximately $30 \mathrm{~m}$, or the ground area encompassing two rows of solar troughs" (Erickson and others, 2015).

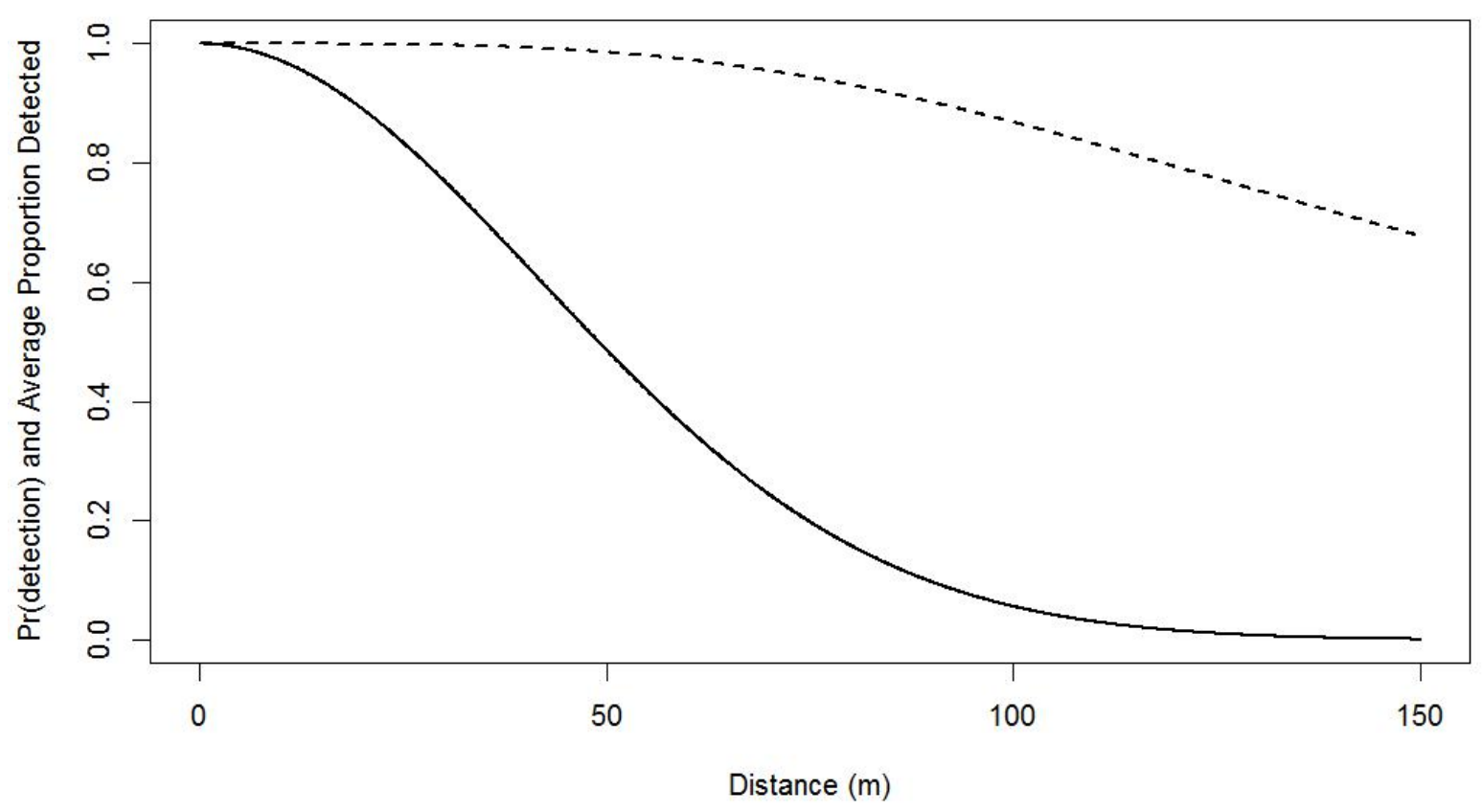

Figure B2. Probability $f(x)$ of observing a carcass $x \mathrm{~m}$ from the observer $(-)$ represented here is a half-normal with standard deviation of 41.7, and standardized to a value of 1 at $x=0$; and average probability of $\pi_{2}(x)$ of observing a carcass between two transects $x$ meters apart when searchers look to both sides.

Although they do not fit an observer-detection function to their observations, a half-normal detection function with standard deviation of 41.7 (fig. B2, solid line) results in an average observerdetection probability of 0.92 within $30 \mathrm{~m}$ with observers looking only to one side (appendix A) and gives a reasonable representation of a detection function that could be associated with their data. 

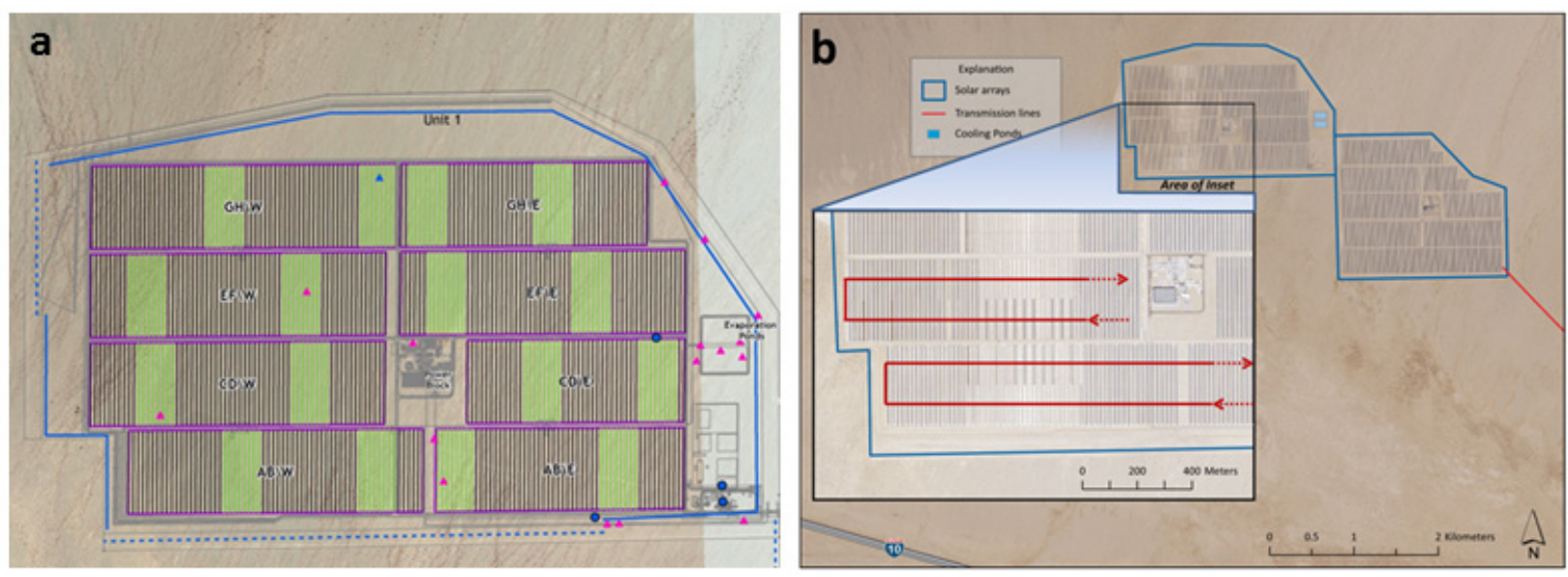

Figure B3. (a) Reproduction of figure 2 from Erickson and others (2015) showing searched area representing 30\% of total area beneath solar troughs at Genesis. Reproduced with permission from Erickson and others (2015). (b) Representation of suggested distance-based search path beneath solar troughs at Genesis.

The total driving distance needed to complete a search of $30.4 \%$ of the site using the meandering method was approximately ${ }^{3} 102 \mathrm{~km}$. In contrast, with only small changes to their protocol, observerdetection could be greatly increased at very little, if any, additional cost. At this facility, joints where pipes passing through the focus of the parabola carrying superheated oil connect to the main oil artery must be checked by technical staff twice a day. These joints occur every $150 \mathrm{~m}$ along each row of mirrors. The technician drives slowly $(<8 \mathrm{kph})$ back and forth below the mirror rows at $150 \mathrm{~m}$ intervals, covering the entire area in less than 4 hours. The entire facility is checked every 12 hours by a single technician. A similar pattern could easily be executed in the carcass search protocol resulting in 100\% coverage of the site with transects separated by $150 \mathrm{~m}$ and the driver looking to both sides (fig. B3b). The total driving distance needed to complete a search of $100 \%$ of the site using the distance-based method would be ${ }^{3} 36 \mathrm{~km}, 65 \%$ less than the $30.4 \%$ sample. However, the average observer-detection probability for small birds over the entire facility would be 0.68 (fig. B2, dashed line) versus 0.28 achieved using the meandering transects in a random sample of $30.4 \%$ of the area. Detection rate is more than doubled yet total effort is reduced by $65 \%$. Removing the $30 \%$ sampling constraint will allow detection of larger carcasses to be increased, perhaps close to $100 \%$.

Further cost saving might be realized when the increased observer-detection using $150 \mathrm{~m}$ transects is balanced against search interval to achieve the target overall detection probability needed to meet study objectives. The higher detection rate with distance-based sampling may allow repeat visits to occur less frequently, depending on the scavenging pressure, and result in lower seasonal or annual costs.

\footnotetext{
${ }^{3}$ Unit 1 comprised four blocks of troughs about $2 \mathrm{~km}$ in length, and Unit 2 comprised five blocks of troughs also about $2 \mathrm{~km}$ in length for a total block-edge length of about $18 \mathrm{~km}$. A total of 280 trough rows, each $300 \mathrm{~m}$ long, comprising $30.4 \%$ of the reflector array at the facility, were searched. Each $30 \mathrm{~m}$ pass, the width of two trough rows, was driven twice, first with the driver looking only forward to assure no obstructions, then again looking out $30 \mathrm{~m}$ only to one side of the vehicle. Total transect length was $102 \mathrm{~km}((280 / 2) * 2 * 300+18 \mathrm{~km}$ edge length traversed to access sampling units). In contrast, the total driving distance needed to traverse the entire site with transects spaced $150 \mathrm{~m}$ apart is $36 \mathrm{~km}$ (two passes in each block).
} 


\section{Case Study 3 - 100\% Traverse of Photovoltaic Arrays Collector Field Versus 20\% Sample}

The search protocol at the Desert Sunlight PV facility in Riverside County, California, in spring 2015 was described as "... biologists slowly walked a transect line along the ends of rows of solar panels in a direction perpendicular to the rows, searching ahead and to the [one] side within the array for bird and bat detections. Biologists scanned out to a maximum perpendicular distance of approximately $70 \mathrm{~m}$ from the transect. ... Each array included in the sample was searched by observers walking two transects - one on the west side and one on the east side of the array with observers looking toward the center of the array" (Western Ecosystems Technology, Inc., 2015). They reported a weighted-average probability of observer-detection for $70 \mathrm{~m}$ wide and $140 \mathrm{~m}$ wide transects combined of 0.596 (95\% CI: $0.505-0.671$ ) for small birds within the searched area (Western Ecosystems Technology, Inc., 2015, fig. 5 and table $\mathrm{C}-1$ ) and fit a detection function to their observations that is well described by a halfnormal with standard deviation of 25.3 which results in an average observer-detection of 0.811 between $70 \mathrm{~m}$ transects and 0.453 within $140 \mathrm{~m}$ transects (searching inward only) and a weighted average SE equal to the reported value of 0.596 . However, searches were conducted in sampling units covering $30 \%$ of the area of the PV array field resulting in a combined detection probability for the facility of about $0.18(=0.3 * 0.596)$ (fig. B4a).
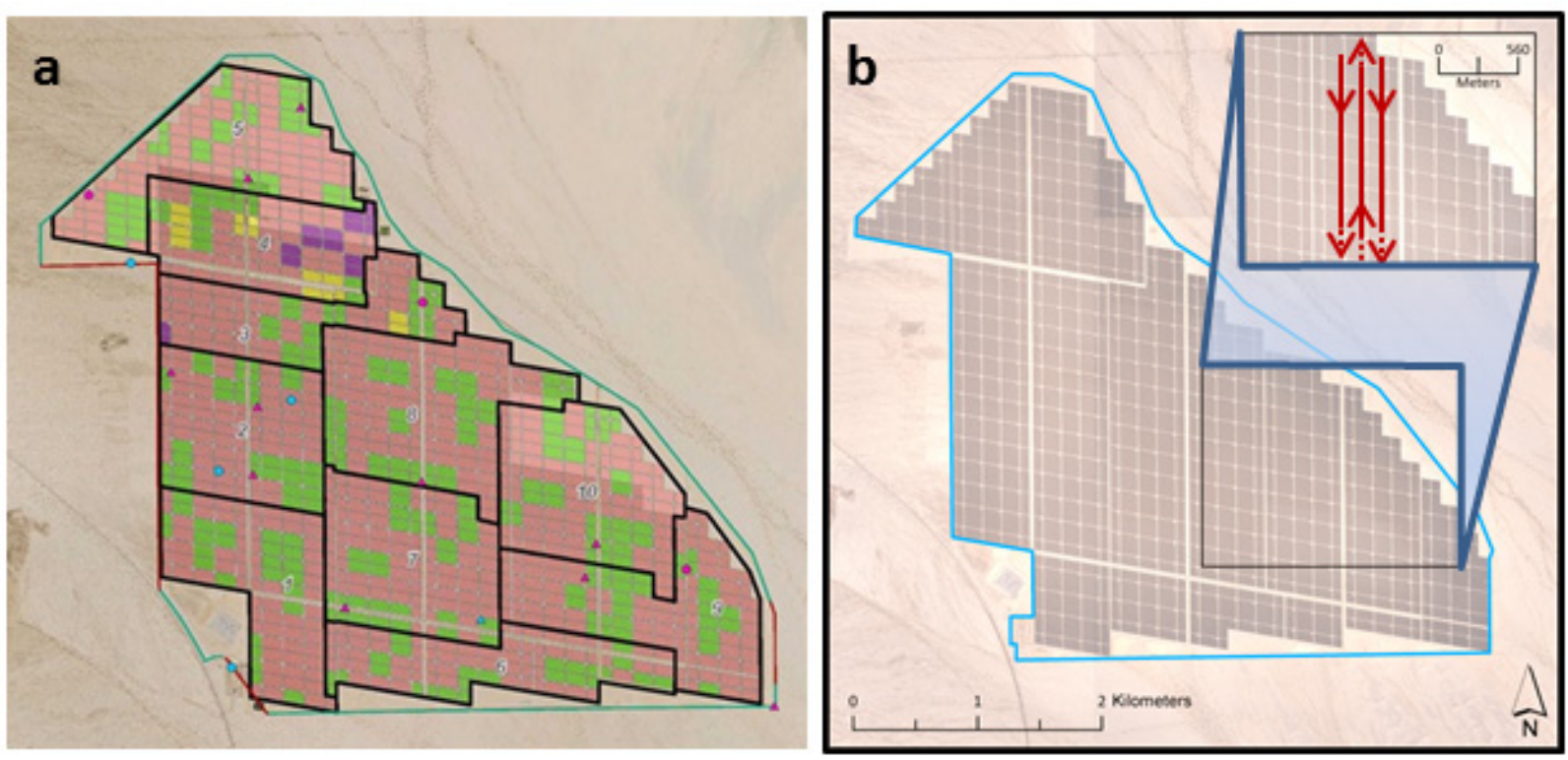

Figure B4. (a) Reproduction of figure 2 from Erickson and others (2015) showing searched area representing 30\% of area of the photovoltaic arrays array field at Desert Sunlight Solar Farm. Reproduced with permission from Erickson and others (2015). (b) Representation of suggested distance-based search path within photovoltaic arrays array at Desert Sunlight Solar Farm. 
An alternative approach would be to traverse the entire site using transects separated by $140 \mathrm{~m}$ (fig. B4b). The total transect length would be $20 \%$ more than that needed to search $30 \%$ of the site ${ }^{4}$. Using the same detection function that they presented, we calculate the average probability of searcher detecting a small bird on the site to be 0.45 , versus 0.18 using a $30 \%$ sample. Thus, an additional $20 \%$ of transect length would more than double the observer-detection rate for small birds.

For medium and large birds within the 30\% sample, the reported average searcher efficiency rates were very high, at 0.872 (95\% CI: $0.786-0.953)$ and 0.977 (95\% CI: $0.931-1.00)$, respectively, but because only $30 \%$ of the site was sampled, site-wide average searcher efficiency rate was only 0.262 and 0.293 , respectively. Removing the sampling fraction constraint would result in site-wide searcher efficiency rates of 0.803 and 0.963 for medium and large birds, respectively.

Cost saving might be realized when the increased searcher efficiency is balanced against search interval to achieve the target overall detection probability needed to meet study objectives. The higher average searcher efficiency rate with distance-based sampling may allow repeat visits to occur less frequently, depending on the scavenging pressure, resulting in lower annual or seasonal costs.

\footnotetext{
${ }^{4}$ Let $\mathrm{T}=$ total distance covered to traverse the entire site along transects separated by $140 \mathrm{~m}$. Then, the total distance needed to traverse a $30 \%$ sample of the site would be $0.3 \mathrm{~T}$. However, in practice $40 \%$ of the sampled area was traversed using transects separated by $70 \mathrm{~m}$ and $60 \%$ using transects separated by $140 \mathrm{~m}$, so the distance needed for a $30 \%$ sample was $(0.3 \mathrm{~T} *(0.4 * 2+0.6)=0.42 \mathrm{~T}$. In addition, each unit was walked twice, once on the east, once on the west with searchers looking inward only, doubling the transect length to $0.84 \mathrm{~T}$. In comparison, the total distance covered to traverse the entire site using transects $140 \mathrm{~m}$ apart and looking in both directions is about $20 \%$ more than the distance covered in the $30 \%$ sample $(1.00 / 0.42)$.
} 


\section{Case Study 4 - 100\% Traverse of Linear Feature Versus 50\% Sample}

At Desert Sunlight Solar Farm in spring 2015, 47.9\% of the $19.2 \mathrm{~km}$ of gen-tie line was surveyed by "[b]iologists slowly walked every other 1-km segment of the line, meandering the width of the strip transect, scanning for dead or injured birds or bats within $15 \mathrm{~m}(49.2 \mathrm{ft})$ of the overhead line." (Western Ecosystems Technology, Inc., 2015, table 1) with a SE rate of 0.54 (Western Ecosystems Technology, Inc., 2015, table C-1). A full search of the gen-tie using this methodology would require approximately 6 hours. In contrast, a searcher could walk the entire length in less time ${ }^{5}$ and by removing the sampling fraction constraint, observer-detection probability for the site would be doubled for all size classes, assuming the SE is the same for both walking transect methods.

\footnotetext{
${ }^{5}$ Let $\mathrm{T}=$ total length of gen-tie, about $20 \mathrm{~km}$. Searchers drive the entire length, stopping every $2 \mathrm{~km}$ to get out, walk $1 \mathrm{~km}$ under the gen-tie, then walk $1 \mathrm{~km}$ back to the vehicle. Total distance covered is $20 \mathrm{~km}$ in vehicle, $20 \mathrm{~km}$ on foot. If vehicle speed limit on the facility is about $20 \mathrm{kph}$ and walking speed is $4 \mathrm{kph}$, this would take approximately 6 hours. In contrast, a searcher walking the full length would cover the same distance in 5 hours.
} 


\section{Case Study 5 - Example Trial Carcass Placement}

We recommend a minimum of 30 bats, 30 small-, 20 medium-, and 15 large-sized bird carcasses be used in SE trials in each season for each feature, and the same number should be used in CP trials. With careful planning, the same carcasses can serve in both trials (table B1). These represent samples sizes for which the ratio of the upper:lower $95 \%$ confidence limits of SEs of $0.25,0.5$, and 0.75 is sixfold, 3-fold, and 2-fold, respectively. They are arbitrary but attempt to balance statistical and economic costs. We emphasize that the recommended trial numbers are minimums for each combination of relevant factors and can and should be increased when feasible to increase precision of fatality estimates, particularly when SE is low. Placing fresh CP and SE trial carcasses regularly throughout the study period will assure that the persistence and observation processes measured in the trials most closely reflect the same processes occurring with actual carcasses. It is very important that the searchers are completely unaware of when and where the SE trial carcasses are being placed, that is, that they are being tested. It is possible, and in some cases desirable, to use the same carcasses for CP and SE trials, but it is critical then, that fresh carcasses be placed regularly throughout the study period, to mimic the carcass arrival process, and not always immediately before a search is conducted in the area. If the same carcasses used for CP trials continue to serve as SE trial carcasses, minimum combined sample sizes should be increased by about $33 \%$ to accommodate attrition due to scavenger removal.

Example: Distributing 65 total bird carcasses (30 small birds, 20 medium birds, and 15 large birds) along a $16.7 \mathrm{~km}$ fence line over a 3-month period.

- Determine locations: $16.7 \mathrm{~km} / 65$ carcasses $\rightarrow 1$ carcass every about $0.25 \mathrm{~km}$.

- Generate random uniform $(-15,15)$ distance from fence: $\pm 15 \mathrm{~m}$ for each $0.25 \mathrm{~km}$ location.

- Randomly assign 10 S, 7 (or 6) M and 5 L to first 22 locations;

- Repeat for next 22, then next 21 locations.

- Calculate placement dates: 65 carcasses/13 weeks $=5$ carcasses per week, 1 each week day.

- Randomly assign placement date to carcass-location. Reorder by date.

Table B1. Example Resulting File:

\begin{tabular}{cccccc}
\hline $\begin{array}{c}\text { Carcass } \\
\text { ID }\end{array}$ & Date & Weekday & Size & $\begin{array}{c}\text { Position } \\
\text { along fence } \\
(\mathbf{k m})\end{array}$ & $\begin{array}{c}\text { Distance } \\
\text { from fence } \\
(\mathbf{m})\end{array}$ \\
\hline 1 & $6 / 22 / 2020$ & $\mathrm{M}$ & $\mathrm{L}$ & 1.50 & 0 \\
2 & $6 / 23 / 2020$ & $\mathrm{~T}$ & $\mathrm{M}$ & 4.00 & -1 \\
3 & $6 / 24 / 2020$ & $\mathrm{~W}$ & $\mathrm{M}$ & 0.50 & -15 \\
4 & $6 / 25 / 2020$ & $\mathrm{H}$ & $\mathrm{S}$ & 7.00 & 3 \\
5 & $6 / 26 / 2020$ & $\mathrm{~F}$ & $\mathrm{M}$ & 13.50 & 13 \\
6 & $6 / 29 / 2020$ & $\mathrm{M}$ & $\mathrm{L}$ & 0.25 & -4 \\
7 & $6 / 30 / 2020$ & $\mathrm{~T}$ & $\mathrm{~S}$ & 2.75 & -7 \\
8 & $7 / 1 / 2020$ & $\mathrm{~W}$ & $\mathrm{~S}$ & 14.50 & 19 \\
$\vdots$ & & & & & \\
62 & $9 / 15 / 2020$ & $\mathrm{~T}$ & $\mathrm{M}$ & 1.75 & 7 \\
63 & $9 / 16 / 2020$ & $\mathrm{~W}$ & $\mathrm{~S}$ & 11.00 & -18 \\
64 & $9 / 17 / 2020$ & $\mathrm{H}$ & $\mathrm{L}$ & 12.75 & 16 \\
65 & $9 / 18 / 2020$ & $\mathrm{~F}$ & $\mathrm{~S}$ & 11.25 & $\vdots$ \\
\hline
\end{tabular}


For SE: Note whether an observer detected the carcass on a scheduled search. If a searcher fails to detect a carcass, the crew leader must check to make sure it was still present and available to be detected before marking it as missed. Leave the carcass in place and continue to note in the same manner whether an observer detected the carcass on each subsequent scheduled search until it has been found or is no longer present. See example data sheet in appendix A.

For CP: Note time and date of placement. Check each trial carcass at least daily for the first 4 days decreasing frequency of checks as the carcass persists. The following check schedule: Day 1,2,3, $4,7,10,14,17,21,28$, fits well with conventional 5-day work weeks. At each check, note time and date and whether the carcass is present (that is, recognizable as a carcass to an observer) or absent, that is, either removed or no longer recognizable as a carcass by a searcher. See example data sheet in appendix A. Alternatively, camera traps can be used to determine the exact date and time that a carcass is removed, but should be checked regularly to assure proper functioning throughout the monitoring period. The CP trial ends on the 28th day when any remaining carcasses should be removed by the crew leader. Persistence models will be fit using survival analysis models, allowing the potential for nonconstant removal rates in time. 


\section{Appendix C. Example Data}

Table C1. Observed carcasses.

\begin{tabular}{|c|c|}
\hline Column Name & Description \\
\hline CarcassID & yyyymmddSPCD\#\# \\
\hline Searcher & 3-letter initials or unambiguous short identifier \\
\hline Feature & Inner heliostat, outer heliostat, $\mathrm{PV}, \mathrm{ST}$, pond, building, fence, tr-line \\
\hline Unit & Local reference system for identifying location \\
\hline Transect\# & Local reference system for identifying location \\
\hline UTM Zone & UTM Zone for reference \\
\hline UTME & UTM Easting of the carcass \\
\hline UTMN & UTM Northing of the carcass \\
\hline DistanceFromSearcher & Distance from searcher to carcass $(\mathrm{m})$ at first detection \\
\hline PerpDistFromTransect & Perpendicular (minimum) distance to carcass from transect line $(\mathrm{m})$ \\
\hline DateFound & yyyy/mm/dd hh:mm \\
\hline DatePrevSearch & yyyy/mm/dd hh:mm \\
\hline Season & $\mathrm{Sp}, \mathrm{Su}, \mathrm{Fa}, \mathrm{Wi}$ or alternative applicable code \\
\hline VisClass & vegetated, open \\
\hline Shade & Shade, NoShade \\
\hline Bird.Bat & Bird, Bat \\
\hline Guild & General behavioral guild or group, for example, raptor, passerine, shorebird \\
\hline CommonName & Common name for species, if identifiable, UNKN if not \\
\hline Species & Scientific name for species, if identifiable, UNKN if not \\
\hline SpCode & 4-letter unambiguous species code \\
\hline CarcassSize (g) & average weight of species/gender in grams \\
\hline Age & Adult, juvenile, Unknown \\
\hline Sex & M, F, U (unknown) \\
\hline Live/Dead & L (live), D (dead) \\
\hline Released/Euthanized & R, E, D (dead) \\
\hline $\begin{array}{l}\text { EstimatedTimeSinceDeath or } \\
\text { Injury }\end{array}$ & $0.5(<12$ hours $), 1(<24$ hours $), 2(<$ days $)$, etc. \\
\hline Incidental & Was carcass found on scheduled search? \\
\hline CauseofDeath/Injury & Collision, Heat, Singeing, Other, Unknown \\
\hline CertaintyofCause & $\begin{array}{l}\% \text { certainty in assignment of cause of death; } 10 \% \text { increments from } 0 \% \text { (unknown } \\
\text { cause) }-100 \% \text { (fully known cause) }\end{array}$ \\
\hline Photo\# & Local reference system for identifying photo of carcass \\
\hline
\end{tabular}


Table C2. Searcher efficiency and carcass persistence trial carcasses.

\begin{tabular}{|c|c|}
\hline Column Name & Description \\
\hline CarcassID & yyyymmddSPCD\#\# \\
\hline Unit & Local reference system for identifying location \\
\hline Feature & Inner heliostat, outer heliostat, $\mathrm{PV}, \mathrm{ST}$, pond, building, fence, tr-line \\
\hline Transect\# & Local reference system for identifying location \\
\hline UTM Zone & UTM Zone for reference \\
\hline UTME & UTM Easting of the carcass \\
\hline UTMN & UTM Northing of the carcass \\
\hline DistanceFromSearcher & Distance from searcher to carcass $(\mathrm{m})$ at first detection \\
\hline PerpDistFromTransect & Perpendicular (minimum) distance to carcass from transect line $(\mathrm{m})$ \\
\hline DatePlaced & yyyy/mm/dd hh:mm \\
\hline Season & $\mathrm{Sp}, \mathrm{Su}, \mathrm{Fa}, \mathrm{Wi}$ or alternative applicable code \\
\hline VisClass & vegetated, open \\
\hline Shade & Shade, NoShade \\
\hline Bird.Bat & Bird, Bat \\
\hline Guild & General behavioral guild or group, for example, raptor, passerine, shorebird \\
\hline CommonName & Common name for species, if identifiable, UNKN if not \\
\hline Species & Scientific name for species, if identifiable, UNKN if not \\
\hline SpCode & 4-letter unambiguous species code \\
\hline CarcassSize (g) & average weight of species/gender in grams \\
\hline Age & Adult, juvenile, Unknown \\
\hline Sex & M, F, U (unknown) \\
\hline EstimatedTimeSinceDeath & 0.5 ( $<12$ hours), $1(<24$ hours), $2(<$ days $)$, etc. \\
\hline Date1stSearch & yyyy/mm/dd hh:mm \\
\hline Found1stSearch & $\mathrm{Y}, \mathrm{N}$ \\
\hline Date2ndSearch & yyyy/mm/dd hh:mm \\
\hline Found2ndSearch & $\mathrm{Y}, \mathrm{N}$ \\
\hline Date3rdSearch & yyyy/mm/dd hh:mm \\
\hline Found3rdSearch & $\mathrm{Y}, \mathrm{N}$ \\
\hline Date4thSearch* & yyyy/mm/dd hh:mm \\
\hline Found4thSearch* & $\mathrm{Y}, \mathrm{N}$ \\
\hline Day1 & Intact, Partially scavenged, Feather Spot, Absent \\
\hline Day2 & Intact, Partially scavenged, Feather Spot, Absent \\
\hline Day3 & Intact, Partially scavenged, Feather Spot, Absent \\
\hline Day4 & Intact, Partially scavenged, Feather Spot, Absent \\
\hline Day7 & Intact, Partially scavenged, Feather Spot, Absent \\
\hline Day10 & Intact, Partially scavenged, Feather Spot, Absent \\
\hline Day14 & Intact, Partially scavenged, Feather Spot, Absent \\
\hline Day17 & Intact, Partially scavenged, Feather Spot, Absent \\
\hline Day21 & Intact, Partially scavenged, Feather Spot, Absent \\
\hline Day28 & Intact, Partially scavenged, Feather Spot, Absent \\
\hline
\end{tabular}

*More columns can be added if more searches are conducted. 


\section{Appendix D. Summary Methods}

We recommend transects be situated throughout the full extent of the feature being monitored. Suggested spacing between transects and initial search interval are given as starting points that can be modified as knowledge accumulates regarding actual persistence patterns and searcher efficiency with distance.

Table D1. Search methods for features at Power Tower facilities.

\begin{tabular}{|c|c|c|c|c|}
\hline Feature & $\begin{array}{l}\text { Transect } \\
\text { width }\end{array}$ & $\begin{array}{l}\text { Search } \\
\text { interval }\end{array}$ & Search pattern & Transportation \\
\hline Central circle & $20-25 \mathrm{~m}$ & 3-4 days & $\begin{array}{l}\text { Along natural lines formed } \\
\text { by support structures }\end{array}$ & Foot, ATV, small truck \\
\hline Outer circle & $50 \mathrm{~m}$ & Weekly & $\begin{array}{l}\text { Along circular access roads } \\
\text { throughout field }\end{array}$ & Foot, ATV, small truck \\
\hline Buildings & variable & Continuous & Random & Foot \\
\hline Ponds & variable & 3-4 days & Along perimeter of pond & Foot \\
\hline Gen-ties & variable & Weekly & Single transect below lines & Foot, ATV, small truck \\
\hline Fences & variable & Weekly & $\begin{array}{l}\text { Single transect along fence } \\
\text { within facility }\end{array}$ & Foot, ATV, small truck \\
\hline
\end{tabular}

Table D2. Search methods for features at Solar Trough facilities.

\begin{tabular}{lllll}
\hline \multicolumn{1}{c}{ Feature } & $\begin{array}{c}\text { Transect } \\
\text { width }\end{array}$ & $\begin{array}{c}\text { Search } \\
\text { interval }\end{array}$ & \multicolumn{1}{c}{ Search pattern } & \multicolumn{1}{c}{ Transportation } \\
\hline Reflector field & $150 \mathrm{~m}$ & $0.5-1$ day & $\begin{array}{c}\text { Perpendicular to major axis } \\
\text { of mirror rows; mid-way } \\
\text { between row-ends }\end{array}$ & ATV, small truck \\
Buildings & variable & $3-4$ days & $\begin{array}{l}\text { Random } \\
\text { Along perimeter of pond }\end{array}$ & Foot \\
Ponds & variable & Weekly & $\begin{array}{l}\text { Single transect below lines } \\
\text { Gen-ties }\end{array}$ & Foot, ATV, small truck \\
Fences & variable & Weekly & $\begin{array}{l}\text { Single transect along fence } \\
\text { within facility }\end{array}$ & Foot, ATV, small truck \\
\hline
\end{tabular}

Table D3. Search methods for features at Photovoltaic facilities.

\begin{tabular}{|c|c|c|c|c|}
\hline Feature & $\begin{array}{l}\text { Transect } \\
\text { width }\end{array}$ & $\begin{array}{l}\text { Search } \\
\text { interval }\end{array}$ & Search pattern & Transportation \\
\hline Collector Field & $280 \mathrm{~m}$ & Weekly & $\begin{array}{l}\text { Along access roads } \\
\text { perpendicular to PV rows }\end{array}$ & Foot, ATV, small truck \\
\hline Buildings & & Continuous & Random & Foot \\
\hline Gen-ties & variable & Weekly & Single transect below lines & Foot, ATV, small truck \\
\hline Fences & variable & Weekly & $\begin{array}{l}\text { Single transect along fence } \\
\text { within facility }\end{array}$ & Foot, ATV, small truck \\
\hline
\end{tabular}


Publishing support provided by the U.S. Geological Survey Science Publishing Network, Tacoma Publishing Service Center

For more information concerning the research in this report, contact the Director, Forest and Rangeland Ecosystem Science Center U.S. Geological Survey

777 NW 9th St., Suite 400

Corvallis, Oregon 97330

http://fresc.usgs.gov/ 
웅

$\frac{\pi}{6}$

을

(1)

을

D

嘼

궁

홀

?

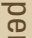

高

ग्ञ

응

음

i 